TRANSACTIONS OF THE

AMERICAN MATHEMATICAL SOCIETY

Volume 363, Number 5, May 2011, Pages 2571-2598

S 0002-9947(2010)05148-7

Article electronically published on December 20, 2010

\title{
DIFFUSIVE STABILITY OF OSCILLATIONS IN REACTION-DIFFUSION SYSTEMS
}

\author{
THIERRY GALLAY AND ARND SCHEEL
}

\begin{abstract}
We study nonlinear stability of spatially homogeneous oscillations in reaction-diffusion systems. Assuming absence of unstable linear modes and linear diffusive behavior for the neutral phase, we prove that spatially localized perturbations decay algebraically with the diffusive rate $t^{-n / 2}$ in space dimension $n$. We also compute the leading order term in the asymptotic expansion of the solution and show that it corresponds to a spatially localized modulation of the phase. Our approach is based on a normal form transformation in the kinetics ODE which partially decouples the phase equation at the expense of making the whole system quasilinear. Stability is then obtained by a global fixed point argument in temporally weighted Sobolev spaces.
\end{abstract}

\section{INTRODUCTION AND MAIN RESULTS}

Synchronization of spatially distributed dissipative oscillators has been observed in a wide variety of physical systems. We mention synchronization in yeast cell populations [14, fireflies 4], coupled laser arrays [26], and spatially homogeneous oscillations in reaction-diffusion systems such as the Belousov-Zhabotinsky reaction 32 and the NO+CO-reaction on a $\mathrm{Pt}(100)$ surface [31. Synchronization strikes us most when the system size is large or the coupling strength is weak. Both situations relate in natural ways to the regime of a large Reynolds number in fluid experiments, where one expects turbulent, incoherent rather than laminar, synchronized behavior. Still, one finds synchronization as a quite common, universal phenomenon, even in very large systems.

The aim of this article is to elucidate the robustness of spatially homogeneous temporal oscillations in spatially extended systems, under most general assumptions, without detailed knowledge of internal oscillator dynamics or coupling mechanisms. In fact, quantitative models are very rarely available for the systems mentioned above. Instead, we make phenomenological assumptions, related to the existence of oscillations and the absence of strongly unstable modes. These assumptions typically guarantee asymptotic stability of a spatially homogeneous oscillation in any finite-size system, when equipped with compatible (say, Neumann) boundary conditions. The results in this article are concerned with infinite-size, reactiondiffusion systems,

$$
u_{t}=D \Delta u+f(u), \quad u=u(t, x) \in \mathbb{R}^{N}, \quad x \in \mathbb{R}^{n}, \quad t \geq 0,
$$

Received by the editors June 24, 2008 and, in revised form, June 26, 2009.

2010 Mathematics Subject Classification. Primary 35B35, 35B40, 35B10, 35K57.

(C)2010 American Mathematical Society

Reverts to public domain 28 years from publication 
with positive coupling matrix $D \in \mathcal{M}_{N \times N}(\mathbb{R}), D=D^{\top}>0$, and smooth kinetics $f \in C^{\infty}\left(\mathbb{R}^{N}, \mathbb{R}^{N}\right)$. In this spatially continuous setup, working in the whole space $\mathbb{R}^{n}$ is an idealization which corresponds to the limit of a small coupling matrix and/or large domain size. We will briefly comment below on the relation between our results in the whole space and the stability of temporal oscillations in finite domains.

To be specific, we make the following assumptions on the kinetics $f$ and the coupling matrix $D$.

Hypothesis 1.1 (Oscillatory kinetics). We suppose that the ODE $u_{t}=f(u)$ possesses a periodic solution $u_{*}(t)=u_{*}(t+T)$ with minimal period $T>0$.

In particular, to avoid trivial situations, we assume that the periodic orbit is not reduced to a single equilibrium. As is well known, this is possible only if $N \geq 2$, i.e. if system (10) does not reduce to a scalar equation.

In addition to existence we will make a number of assumptions on the Floquet exponents of the linearized equation

$$
u_{t}=D \Delta u+f^{\prime}\left(u_{*}(t)\right) u,
$$

which is formally equivalent (via Fourier analysis) to the family of ordinary differential equations

$$
u_{t}=-k^{2} D u+f^{\prime}\left(u_{*}(t)\right) u, \quad k \in \mathbb{R}^{n} .
$$

For each fixed $k$ we denote by $F_{k}(t, s)$ the two-parameter evolution operator associated to the linear time-periodic system (3), so that $u(t)=F_{k}(t, s) u(s)$ for any $t \geq s$. The asymptotic behavior of the solutions of (3) is well characterized by the Floquet multipliers of the system, that is, the eigenvalues of the period map $F_{k}(T, 0)$. We shall rather work with the Floquet exponents $\lambda_{1}(k), \ldots, \lambda_{N}(k) \in \mathbb{C} / \mathrm{i} \omega \mathbb{Z}$, where $\omega=2 \pi / T$, which satisfy

$$
\operatorname{det}\left(F_{k}(T, 0)-\mathrm{e}^{\lambda_{j}(k) T}\right)=0, \quad j=1, \ldots, N .
$$

Observe that any Floquet exponent which is simple (that is, of algebraic multiplicity one) depends smoothly on the parameter $k$. Also note that $\lambda_{1}=0$ is always a Floquet exponent for $k=0$. We refer to the set of Floquet exponents as the Floquet spectrum.

Hypothesis 1.2 (Marginally stable spectrum). We suppose that the Floquet spectrum in the closed half-space $\{\operatorname{Re} \lambda \geq 0\}$ is minimal. More precisely, we assume that

(i) The Floquet spectrum in the closed half-space $\{\operatorname{Re} \lambda \geq 0\}$ is nonempty only for $k=0$, in which case it consists of a simple Floquet exponent $\lambda_{1}=0$.

(ii) Near $k=0$, the neutral Floquet exponent continues as $\lambda_{1}(k)=-d_{0} k^{2}+\mathrm{O}\left(k^{4}\right)$ for some $d_{0}>0$.

We emphasize that these assumptions are satisfied for an open class of reactiondiffusion systems. In particular the expansion (ii) with some $d_{0} \in \mathbb{R}$ is a consequence of the simplicity of the Floquet exponent $\lambda_{1}=0$ at $k=0$, and of the fact that (3) depends only on $k^{2}$ (and not on $k$ itself). Assuming $d_{0}>0$ is therefore robust. In fact, it is not difficult to show that

$$
d_{0}=\frac{\int_{0}^{T}\left(U_{*}(t), D u_{*}^{\prime}(t)\right) \mathrm{d} t}{\int_{0}^{T}\left(U_{*}(t), u_{*}^{\prime}(t)\right) \mathrm{d} t},
$$


where $(\cdot, \cdot)$ denotes the usual scalar product in $\mathbb{R}^{N}$, and $U_{*}(t)$ is the (unique nontrivial) bounded solution of the adjoint equation

$$
-U_{t}=f^{\prime}\left(u_{*}(t)\right)^{\top} U
$$

Of course, a necessary condition for Hypothesis 1.2 to hold is that $u_{*}(t)$ be a stable periodic solution of the ODE $u_{t}=f(u)$, but this assumption alone is not sufficient in general, except if the diffusion matrix is a multiple of the identity. Indeed, even if $N=2$, one can find examples of periodic solutions which are asymptotically stable for the ODE dynamics, but become unstable if a suitable diffusion is added [23, 24, 25]. One possible scenario, which is usually called phase instability or sideband instability, is that the coefficient $d_{0}$ be negative, in which case the periodic orbit is unstable with respect to long-wavelength perturbations. It may also happen that the Floquet spectrum is stable for $k$ in a neighborhood of the origin, but that there exists an unstable Floquet exponent for some $k_{*} \neq 0$, and therefore for all $k$ in a neighborhood of $k_{*}$. This mechanism is reminiscent of the Turing instability for spatially homogeneous equilibria. In Section 7 below, we give an example of a simple 2-species system which exhibits both kinds of instabilities depending on the choice of the parameters.

In order to state our results, we introduce a function space which measures both the spatial localization and the amplitude of the perturbation to our spatially homogeneous profile $u_{*}$. We will consider initial perturbations in the space of functions $X=L^{1}\left(\mathbb{R}^{n}\right) \cap L^{\infty}\left(\mathbb{R}^{n}\right)$, with target space $\mathbb{R}^{N}$, equipped with the norm

$$
\|v\|_{X}=\int_{\mathbb{R}^{n}}|v(x)| \mathrm{d} x+\sup _{x \in \mathbb{R}^{n}}|v(x)|,
$$

where "sup" here refers to the essential supremum. We will measure decay in the space $L^{\infty}\left(\mathbb{R}^{n}\right)$. Our first result is:

Theorem 1.3. Consider a reaction-diffusion system (1) on $\mathbb{R}^{n}$ with oscillatory kinetics, Hypothesis 1.1, and marginally stable spectrum, Hypothesis 1.2. Then there are $C, \delta>0$ such that for any initial data $u(0, x)=u_{*}\left(t_{0}\right)+v_{0}(x)$ with $t_{0} \in \mathbb{R}$ arbitrary and $\left\|v_{0}\right\|_{X} \leq \delta$, there exists a unique, smooth global solution $u(t, x)$ of (11) for $t \geq 0$. Moreover $u(t, x)$ converges to the periodic solution $u_{*}$ in the sense that

$$
\sup _{x \in \mathbb{R}^{n}}\left|u(t, x)-u_{*}\left(t_{0}+t\right)\right| \leq \frac{C\left\|v_{0}\right\|_{X}}{(1+t)^{n / 2}}, \quad \text { for all } t \geq 0 .
$$

We emphasize that the perturbations we consider are localized in space and therefore do not alter the overall phase $t_{0}$ of the periodic solution. We refer however to Section 7 for a discussion of possible stability results in more general situations. It is not difficult to verify that the decay rate in (6) is optimal. In fact, under the assumptions of Theorem 1.3. one can even compute the leading term in the asymptotic expansion of the perturbation as $t \rightarrow+\infty$. Let

$$
G(x)=\frac{1}{\left(4 \pi d_{0}\right)^{n / 2}} \exp \left(-\frac{|x|^{2}}{4 d_{0}}\right), \quad x \in \mathbb{R}^{n},
$$

where $d_{0}>0$ is defined in (4). Our second result is:

Theorem 1.4. Under the assumptions of Theorem 1.3, the solution $u(t, x)$ of (1) can be decomposed as

$$
u(t, x)=u_{*}\left(t_{0}+t\right)+u_{*}^{\prime}\left(t_{0}+t\right) \alpha(t, x)+\beta(t, x), \quad x \in \mathbb{R}^{n}, \quad t \geq 0,
$$


where $\alpha: \mathbb{R}_{+} \times \mathbb{R}^{n} \rightarrow \mathbb{R}$ and $\beta: \mathbb{R}_{+} \times \mathbb{R}^{n} \rightarrow \mathbb{R}^{N}$ satisfy

$$
\begin{aligned}
& \|\beta(t, \cdot)\|_{L^{1}}+(1+t)^{n / 2}\|\beta(t, \cdot)\|_{L^{\infty}} \quad \underset{t \rightarrow+\infty}{\longrightarrow} 0, \\
& \left\|t^{n / 2} \alpha(t, x \sqrt{t})-\alpha_{*} G\right\|_{L^{1} \cap L^{\infty}} \quad \underset{t \rightarrow+\infty}{\longrightarrow} 0,
\end{aligned}
$$

for some $\alpha_{*} \in \mathbb{R}$. In addition,

$$
\alpha_{*}=\int_{\mathbb{R}^{n}} \frac{\left(U_{*}\left(t_{0}\right), v_{0}(x)\right)}{\left(U_{*}\left(t_{0}\right), u_{*}^{\prime}\left(t_{0}\right)\right)} \mathrm{d} x+\mathrm{O}\left(\delta^{2}\right),
$$

where $U_{*}(t)$ is the bounded solution of the adjoint equation (5).

In other words, the solution $u(t, x)$ of (1) satisfies

$$
\begin{aligned}
u(t, x) & =u_{*}\left(t_{0}+t\right)+u_{*}^{\prime}\left(t_{0}+t\right) \frac{\alpha_{*}}{\left(4 \pi d_{0} t\right)^{n / 2}} \mathrm{e}^{-|x|^{2} /\left(4 d_{0} t\right)}+\mathrm{o}\left(t^{-n / 2}\right) \\
& =u_{*}\left(t_{0}+t+\frac{\alpha_{*}}{\left(4 \pi d_{0} t\right)^{n / 2}} \mathrm{e}^{-|x|^{2} /\left(4 d_{0} t\right)}\right)+\mathrm{o}\left(t^{-n / 2}\right), \quad \text { as } t \rightarrow+\infty .
\end{aligned}
$$

To leading order, the effect of the perturbation is thus a spatially localized modulation of the phase of the periodic solution. As is clear from the proof, the left-hand side of (9) decays at least like $t^{-\gamma}$ as $t \rightarrow \infty$, for some $\gamma>0$. However, to specify a convergence rate in (10), it is necessary to restrict ourselves to more strongly localized perturbations. For instance, if we assume in addition that $(1+|x|) v_{0} \in L^{1}\left(\mathbb{R}^{n}\right)$, then we can prove that the left-hand side of (10) is $\mathrm{O}\left(t^{-1 / 2}\right)$ as $t \rightarrow \infty$.

Under Hypothesis 1.2 if we consider system (11) in a large bounded domain (say $x \in \Omega / \varepsilon$, where $\Omega \subset \mathbb{R}^{n}$ is bounded) with Neumann boundary conditions, the perturbations of the periodic solution $u_{*}(t)$ decay exponentially [15]: if $\left\|v_{0}\right\|_{L^{\infty}} \leq \delta$ for some small $\delta>0$, then $\|v(t)\|_{L^{\infty}} \leq C\left\|v_{0}\right\|_{L^{\infty}} \mathrm{e}^{-\mu t}$ for some $\mu>0$. However, the relaxation rate $\mu$ and the size of admissible perturbations $\delta$ both depend on $\varepsilon$, with typical scalings $\mu, \delta=\mathrm{O}\left(\varepsilon^{2}\right)$ predicted by the spectral gap of the Laplacian on the domain $\Omega / \varepsilon$. This gap vanishes in the limit $\varepsilon \rightarrow 0$, and Theorem 1.3 shows that exponential decay is replaced by diffusive decay. Nevertheless, we expect our results to give an accurate description of the intermediate asymptotics for large bounded domains if the initial perturbations are sufficiently localized.

The type of diffusive decay that we establish in Theorems 1.3 and 1.4 has been observed in many other contexts. For instance, localized perturbations of spatially periodic, stationary patterns in the Ginzburg-Landau or Swift-Hohenberg equation exhibit a similar diffusive behavior [2, 6, 7, 17, 28, 30. At a technical level, the approach in [2, 28, 30] is based on renormalization group theory; see for instance 3. Roughly speaking, the method relies on the fact that the time- $T$ map for the evolution of the perturbations becomes a contraction in a space of localized functions when composed with an appropriate rescaling, except for a neutral direction which specifies the profile of the self-similar solution describing the leading order asymptotics. A nice feature of renormalization theory is that it allows us to determine easily which terms in the nonlinearity are "relevant" (that is, potentially dangerous) for the stability analysis. For example, if we consider the nonlinear heat equation $u_{t}=\Delta u+|u|^{p}$ in $\mathbb{R}^{n}$, with small and localized initial data, it is well known that the nonlinearity $|u|^{p}$ will not influence the decay predicted by the linear evolution if $p>1+2 / n$, whereas instabilities and even blow-up phenomena can occur if $p \leq 1+2 / n$ [12, 7]. In particular, quadratic terms (which arise naturally in the Taylor expansion of any smooth function) are "irrelevant" if $n \geq 3$ and "relevant" 
if $n=1,2$. For this reason, diffusive stability is often easier to establish in high space dimensions, when diffusion is strong enough to control all possible nonlinear terms, whereas serious problems can occur in low dimensions. This is the case in particular in the stability analysis of one-dimensional spatially periodic patterns [28, 10], where a key step of the proof is to show that relevant "self-coupling" terms actually do not occur in the evolution equation for the neutral translational mode.

As one may expect from the discussion above, Theorem 1.3 is rather easy to prove when $n \geq 3$. For completeness, we first settle this case in Section 2 and then focus on the more interesting situation where $n=1$ or 2 . Here the idea is to construct a normal form transformation for the ODE dynamics which removes all "relevant" terms in the nonlinear PDE satisfied by the perturbation. In Section 3, we show that this is possible, at the expense of transforming the semilinear equation (11) into a quasilinear parabolic system. The next important step is to obtain optimal decay estimates for the solutions of the linearized perturbation equation, including maximal regularity estimates, using the spectral assumptions in Hypothesis 1.2 Since the perturbation equation is translation-invariant in space and periodic in time, such bounds are relatively straightforward to obtain via Fourier analysis; see Section 4. Using these linear estimates, we give in Section 5 a proof of Theorem 1.3 which is valid for $n \leq 3$, hence covering the missing cases $n=1,2$. Instead of renormalization group theory, we prefer using a global fixed point argument in temporally weighted spaces, as in 6, 7, 17. After stability has been established, a rather classical procedure, which is recalled in Section 6, allows us to derive the first-order asymptotics and to prove Theorem 1.4 at least for $n \leq 3$ (the higher-dimensional case is again easier, and left to the reader). In the final section, Section 7 we illustrate our spectral assumptions on a simple, explicit example, and we conclude with a short discussion including possible extensions of our results.

\section{Stability in high Dimensions}

In this section we explore a straightforward and somewhat naive approach to the stability of the periodic orbit $u_{*}(t)$ as a solution of the reaction-diffusion system (11). This method gives a simple proof of Theorem [1.3 in the high-dimensional case $n \geq 3$, the main ingredient of which is an $L^{p}-L^{q}$ estimate for the linearized evolution operator which will be established in Section 4 . Without loss of generality, we assume from now on that the parameter $t_{0}$ in Theorems 1.3 and 1.4 is equal to zero (this is just an appropriate choice of the origin of time).

Consider a solution $u(t, x)=u_{*}(t)+v(t, x)$ of (11). The perturbation $v$ satisfies the equation

$$
v_{t}=D \Delta v+f^{\prime}\left(u_{*}(t)\right) v+N\left(u_{*}(t), v\right)
$$

where

$$
N\left(u_{*}(t), v\right)=f\left(u_{*}(t)+v\right)-f\left(u_{*}(t)\right)-f^{\prime}\left(u_{*}(t)\right) v .
$$

The Cauchy problem for the semi-linear parabolic system (12) is locally well-posed in the space $X=L^{1}\left(\mathbb{R}^{n}\right) \cap L^{\infty}\left(\mathbb{R}^{n}\right)$; see e.g. [15, 20]. More precisely, for any $v_{0} \in X$, there exists a time $\tilde{T}>0$ (depending only on $\left\|v_{0}\right\|_{X}$ ) such that (12) has a unique (mild) solution $v \in C^{0}\left([0, \tilde{T}], L^{1}\left(\mathbb{R}^{n}\right)\right) \cap C_{b}^{0}\left((0, \tilde{T}], L^{\infty}\left(\mathbb{R}^{n}\right)\right)$ satisfying $v(0)=v_{0}$.

Remark 2.1. Due to parabolic regularization, the solution $v(t, x)$ of (12) is smooth for $t>0$. For instance, there exists $C>0$ such that $\|v(t)\|_{H^{2}} \leq C t^{-1}\left\|v_{0}\right\|_{X}$ for all 
$t \in(0, \tilde{T}]$. Therefore, in the proof of Theorem [1.3, we can assume without loss of generality that the initial perturbation is small in the space $X \cap H^{2}\left(\mathbb{R}^{n}\right)$.

To investigate the long-time behavior of the solutions of (12), we consider the corresponding integral equation

$$
v(t)=\mathcal{F}(t, 0) v_{0}+\int_{0}^{t} \mathcal{F}(t, s) N\left(u_{*}(s), v(s)\right) \mathrm{d} s,
$$

where $\mathcal{F}(t, s)$ is the two-parameter semigroup associated to the linearized equation (2). Due to our spectral assumptions (Hypothesis 1.2), the operator $\mathcal{F}(t, s)$ satisfies the same $L^{p}-L^{q}$ estimates as the heat semigroup $\mathrm{e}^{(t-s) \Delta}$. More precisely, anticipating the results of Section 4 , we have:

Proposition 2.2 ( $L^{p}-L^{q}$ estimates). There exists a positive constant $C$ such that, for all $t>s$ and all $1 \leq p \leq q \leq \infty$, we have

$$
\|\mathcal{F}(t, s) v\|_{L^{q}\left(\mathbb{R}^{n}\right)} \leq \frac{C}{(t-s)^{\frac{n}{2}\left(\frac{1}{p}-\frac{1}{q}\right)}}\|v\|_{L^{p}\left(\mathbb{R}^{n}\right)} .
$$

Proof. The proof follows exactly the same lines as in Propositions $4.1,4.3$ and 4.4.

By construction, the nonlinearity $N\left(u_{*}, v\right)$ in (12) is at least quadratic in $v$ in a neighborhood of the origin. More precisely, there exists a nondecreasing function $K: \mathbb{R}_{+} \rightarrow \mathbb{R}_{+}$such that, for all $t \in[0, T]$,

$$
\left|N\left(u_{*}(t), v\right)\right| \leq K(R)|v|^{2} \quad \text { whenever }|v| \leq R .
$$

As was mentioned in the introduction, if the space dimension $n$ is greater than or equal to 3, the diffusive effect described in (14) is strong enough to kill the potential instabilities due to the nonlinearity. In that case, nonlinear stability can therefore be established by a classical argument, which we briefly reproduce here for the reader's convenience.

Proof of Theorem $1.3(n \geq 3)$. Fix $v_{0} \in X=L^{1}\left(\mathbb{R}^{n}\right) \cap L^{\infty}\left(\mathbb{R}^{n}\right)$, and let $v \in$ $C^{0}\left(\left[0, T_{*}\right), L^{1}\left(\mathbb{R}^{n}\right)\right) \cap C^{0}\left(\left(0, T_{*}\right), L^{\infty}\left(\mathbb{R}^{n}\right)\right)$ be the maximal solution of (12) with initial data $v_{0}$. For $t \in\left[0, T_{*}\right)$ we denote

$$
\phi(t)=\sup _{0 \leq s \leq t}\|v(s)\|_{L^{1}}+\sup _{0 \leq s \leq t}(1+s)^{n / 2}\|v(s)\|_{L^{\infty}} .
$$

Using the integral equation (13) and the linear estimates (14), we easily find

$$
\begin{aligned}
\|v(t)\|_{L^{1}} & \leq\left\|\mathcal{F}(t, 0) v_{0}\right\|_{L^{1}}+\int_{0}^{t}\left\|\mathcal{F}(t, s) N\left(u_{*}(s), v(s)\right)\right\|_{L^{1}} \mathrm{~d} s \\
& \leq C\left\|v_{0}\right\|_{L^{1}}+C K(\phi(t)) \int_{0}^{t}\|v(s)\|_{L^{1}}\|v(s)\|_{L^{\infty}} \mathrm{d} s \\
& \leq C\left\|v_{0}\right\|_{L^{1}}+C K(\phi(t)) \phi(t)^{2} \int_{0}^{t} \frac{1}{(1+s)^{n / 2}} \mathrm{~d} s .
\end{aligned}
$$

Similarly, if $0<t<1$, we have

$$
\begin{aligned}
(1+t)^{n / 2}\|v(t)\|_{L^{\infty}} & \leq C\left\|v_{0}\right\|_{L^{\infty}}+C \int_{0}^{t}\left\|N\left(u_{*}(s), v(s)\right)\right\|_{L^{\infty}} \mathrm{d} s \\
& \leq C\left\|v_{0}\right\|_{L^{\infty}}+C K(\phi(t)) \phi(t)^{2} \int_{0}^{t} \frac{1}{(1+s)^{n}} \mathrm{~d} s
\end{aligned}
$$


while for $t \geq 1$ we can bound

$$
\begin{array}{rl}
(1+t)^{n / 2}\|v(t)\|_{L^{\infty}} \leq C\left\|v_{0}\right\|_{L^{1}} & C(1+t)^{n / 2} \int_{0}^{t / 2} \frac{1}{(t-s)^{n / 2}}\left\|N\left(u_{*}(s), v(s)\right)\right\|_{L^{1}} \mathrm{~d} s \\
& +C(1+t)^{n / 2} \int_{t / 2}^{t}\left\|N\left(u_{*}(s), v(s)\right)\right\|_{L^{\infty}} \mathrm{d} s \\
\leq C\left\|v_{0}\right\|_{L^{1}} & +C K(\phi(t)) \phi(t)^{2} \int_{0}^{t} \frac{1}{(1+s)^{n / 2}} \mathrm{~d} s .
\end{array}
$$

Now, since $n \geq 3$, we have $\int_{0}^{\infty}(1+s)^{-n / 2} \mathrm{~d} s<\infty$ and we see that there exist positive constants $C_{1}, C_{2}$ (independent of $T_{*}$ ) such that

$$
\phi(t) \leq C_{1}\left\|v_{0}\right\|_{X}+C_{2} K(\phi(t)) \phi(t)^{2}, \quad \text { for all } t \in\left[0, T_{*}\right) .
$$

So if we further assume that the initial perturbation $v_{0} \in X$ is small enough so that

$$
2 C_{1}\left\|v_{0}\right\|_{X}<1 \text { and } 4 C_{1} C_{2} K(1)\left\|v_{0}\right\|_{X}<1
$$

then it follows from (15) that $\phi(t) \leq 2 C_{1}\left\|v_{0}\right\|_{X}<1$ for all $t \in\left[0, T_{*}\right)$. Since $\left[0, T_{*}\right)$ is the maximal existence interval, this bound implies that $T_{*}=+\infty$ and that the solution of (12) satisfies

$$
\sup _{t \geq 0}\|v(t)\|_{L^{1}}+\sup _{t \geq 0}(1+t)^{n / 2}\|v(t)\|_{L^{\infty}} \leq 2 C_{1}\left\|v_{0}\right\|_{X} .
$$

This concludes the proof of Theorem 1.3 in the high-dimensional case $n \geq 3$.

\section{Reduction to A nORMAL FORM}

In low space dimensions the argument presented in the previous section fails, and we must therefore have a closer look at the structure of the perturbation equation. The idea is to introduce a normal form transformation which simplifies the ODE dynamics in a neighborhood of the periodic orbit $u_{*}$. Applying this transformation to the reaction-diffusion equation (1), we obtain a quasilinear parabolic system which will be the starting point of our stability analysis in Sections 4 and 5

We thus consider the ordinary differential equation

$$
u_{t}=f(u), \quad u \in \mathbb{R}^{N},
$$

with smooth nonlinearity $f \in C^{\infty}\left(\mathbb{R}^{N}, \mathbb{R}^{N}\right)$, and we assume the existence of a time-periodic solution $u_{*}(t)=u_{*}(t+T)$ with minimal period $T=2 \pi / \omega>0$. As in Hypothesis 1.2. we suppose that $u_{*}$ is linearly asymptotically stable, in the sense that the Floquet exponents $\lambda_{1}, \ldots, \lambda_{N}$ are all contained in the open left half-plane, except for $\lambda_{1}=0$ (which is therefore algebraically simple). We shall show that the dynamics of (16) near the periodic orbit $u_{*}$ is conjugate to the dynamics of the following normal form:

$$
\theta_{t}=\omega, \quad \tilde{v}_{t}=g(\theta, \tilde{v}), \quad \theta \in S^{1} \cong \mathbb{R} / 2 \pi \mathbb{Z}, \quad \tilde{v} \in B_{\epsilon} \subset \mathbb{R}^{N-1},
$$

where $B_{\epsilon}$ denotes the open ball of radius $\epsilon>0$ centered at the origin in $\mathbb{R}^{N-1}$. Here the vector field $g$ has the expansion

$$
g(\theta, \tilde{v})=L(\theta) \tilde{v}+g_{2}(\theta, \tilde{v})[\tilde{v}, \tilde{v}],
$$

where $L(\theta)$ is a real $(N-1) \times(N-1)$ matrix depending smoothly on $\theta$, and $g_{2}(\theta, \tilde{v})$ is a symmetric bilinear form on $\mathbb{R}^{N-1}$ depending smoothly on $\theta, \tilde{v}$. In particular $g(\theta, 0)=0$; hence (17) has a trivial solution $\theta(t)=\omega t, \tilde{v}(t)=0$ which 
will correspond to the periodic solution $u_{*}(t)$ of (16). By construction, the Floquet exponents $\lambda_{2}, \ldots, \lambda_{N}$ of the time-periodic linear operator $L(\omega t)$ are all contained in the open left half-plane.

In what follows we denote by $\Phi(t)$ the flow of (16) in a neighborhood of $u_{*}$, and by $\Phi_{\mathrm{nf}}(t)$ the flow of (17) in a neighborhood of $S^{1} \times\{0\}$. These local flows are defined at least for $t \geq 0$.

Lemma 3.1 (Normal form). Assume that the periodic solution $u_{*}$ is linearly asymptotically stable. Then there exist $\epsilon>\epsilon^{\prime}>0$ and a smooth diffeomorphism $\Psi$ from the solid torus $S^{1} \times B_{\epsilon}$ to a tubular neighborhood of the periodic orbit $u_{*}$ such that the local flow in $S^{1} \times \mathbb{R}^{N-1}$ defined on $S^{1} \times B_{\epsilon^{\prime}}$ by

$$
\Phi_{\mathrm{nf}}(t)=\Psi^{-1} \circ \Phi(t) \circ \Psi, \quad t \geq 0,
$$

is the flow induced by an ODE of the form (17), (18).

Proof. Since the periodic orbit $u_{*}$ is linearly asymptotically stable, we can find a tubular neighborhood which is smoothly foliated by strong stable fibers. Straightening out these fibers gives the desired representation of the flow. For completeness we construct this straightening change of coordinates in detail.

We start with the linearized equation at the periodic orbit, $u_{t}=f^{\prime}\left(u_{*}(t)\right) u$ with time evolution $\varphi(t, s)$. We use Floquet theory to construct a linear invariant smooth foliation, first; see [5, §2.4] for some background on Floquet theory. Let $Q$ be the spectral projection for $\varphi(T, 0)$ on span $\left(u_{*}^{\prime}(0)\right)$ and define the $T$-periodic family of projections $Q(t):=\varphi(t, 0) Q \varphi(t, 0)^{-1}$. Then $E^{\mathrm{ss}}(\omega t):=\operatorname{Ker}(Q(t))$ and $E^{\mathrm{c}}(\omega t):=$ $\operatorname{Im}(Q(t))$ form vector spaces with $\operatorname{dim} E^{\mathrm{ss}}(\theta)=N-1$ and $E^{\mathrm{c}}(\theta)=\operatorname{span}\left(u_{*}^{\prime}(\theta / \omega)\right)$. The linearized evolution leaves these subspaces invariant: $u(t) \in E^{\mathrm{ss}}(\theta)$ implies $u(t+\tau) \in E^{\mathrm{ss}}(\theta+\omega \tau)$, and the same holds for $E^{\mathrm{c}}(\theta)$. In particular, the family $\left\{u_{*}(\theta / \omega)+E^{\mathrm{ss}}(\theta)\right\}_{\theta \in S^{1}}$ forms a smooth normal bundle to the periodic orbit $u_{*}$. The normal bundle is trivial since the tangent bundle of the periodic orbit is trivial; a trivialization is given by the linear evolution which induces orientation-preserving maps from $E^{\mathrm{ss}, \mathrm{c}}(0)$ to $E^{\mathrm{ss}, \mathrm{c}}(2 \pi)=E^{\mathrm{ss}, \mathrm{c}}(0)$. Thus, we can find smooth coordinates $(\theta, v) \in S^{1} \times \mathbb{R}^{N-1}$ and a smooth map $\Psi_{0}: S^{1} \times \mathbb{R}^{N-1} \rightarrow \mathbb{R}^{N}$ such that $\Psi_{0}(\theta, 0)=$ $u_{*}(\theta / \omega)$ and $\Psi_{0}\left(\theta, \mathbb{R}^{N-1}\right)=u_{*}(\theta / \omega)+E^{\mathrm{ss}}(\theta)$ for all $\theta \in S^{1}$.

On the other hand, for each $\theta \in S^{1}$, the strong stable manifold $W^{\mathrm{ss}}(\theta)$ of the nonlinear system (16) is the graph of a local map $h_{\theta}: E^{\mathrm{ss}}(\theta) \rightarrow E^{\mathrm{c}}(\theta)$, with $h_{\theta}(0)=0$ and $h_{\theta}^{\prime}(0)=0$. Both strong stable manifolds and their dependence on the base point are smooth; see for instance [11, Theorem 5] which gives arbitrary finite smoothness of stable foliations of center manifolds. In other words, $h_{\theta}$ depends smoothly on $\theta$, so that the map

$$
u_{*}(\theta / \omega)+v^{\mathrm{ss}} \mapsto \Psi_{1}\left(u_{*}(\theta / \omega)+v^{\mathrm{ss}}\right):=u_{*}(\theta / \omega)+v^{\mathrm{ss}}+h_{\theta}\left(v^{\mathrm{ss}}\right)
$$

defines a smooth diffeomorphism in a tubular neighborhood $\mathcal{U}$ of the periodic solution. Thus, if $\epsilon>0$ is sufficiently small, the map $\Psi:=\Psi_{1} \circ \Psi_{0}: S^{1} \times B_{\epsilon} \rightarrow \mathcal{U}$ is also a smooth diffeomorphism onto its image, and $\Psi\left(\theta, B_{\epsilon}\right) \subset u_{*}(\theta / \omega)+W^{\mathrm{ss}}(\theta)$ for all $\theta \in S^{1}$. Since

$$
\Phi(t)\left(u_{*}(\theta / \omega)+W^{\mathrm{ss}}(\theta)\right) \cap \mathcal{U} \subset u_{*}(t+\theta / \omega)+W^{\mathrm{ss}}(\theta+\omega t),
$$

we deduce that, if $\theta \in S^{1}$ and $\tilde{v} \in B_{\epsilon^{\prime}}$ for some small $\epsilon^{\prime}$, then $(\Phi(t) \circ \Psi)(\theta, \tilde{v})$ belongs 
to the image of $\Psi$ for all $t \geq 0$, and

$$
\Phi_{\mathrm{nf}}(t)(\theta, \tilde{v}):=\left(\Psi^{-1} \circ \Phi(t) \circ \Psi\right)(\theta, \tilde{v})=(\theta+\omega t, \hat{v}(t)),
$$

for some $\hat{v}(t) \in B_{\epsilon}$. This immediately implies the trivial form $\theta_{t}=\omega$ for the evolution equation associated to $\Phi_{\mathrm{nf}}$. Moreover, by construction, $\Phi_{\mathrm{nf}}(\theta, 0)=(\theta+$ $\omega t, 0)$ for all $t \geq 0$; hence the transverse variable $\tilde{v}$ evolves according to an ODE of the form (17), where the vector field satisfies $g(\theta, 0)=0$ and can therefore be expanded as in (18).

We conclude this section with the transformation of the full reaction-diffusion system. If we denote $v=(\theta, \tilde{v})^{\top} \in \mathbb{R}^{N}$, the pointwise change of coordinates $u=\Psi(v)$ yields

$$
v_{t}=\Psi^{\prime}(v)^{-1} D \Delta(\Psi(v))+f_{\mathrm{nf}}(v), \quad f_{\mathrm{nf}}(v)=(\omega, g(v))^{\top},
$$

which can be expanded into

$$
v_{t}=\Psi^{\prime}(v)^{-1} D \Psi^{\prime}(v) \Delta v+\Psi^{\prime}(v)^{-1} D \Psi^{\prime \prime}(v)[\nabla v, \nabla v]+f_{\mathrm{nf}}(v) .
$$

We are interested in the stability of the periodic orbit $v_{*}(t)=(\omega t, 0)^{\top}$. We thus set $v=v_{*}(t)+w(t, x)$, so that $w$ solves

$w_{t}=\Psi^{\prime}\left(v_{*}+w\right)^{-1} D \Psi^{\prime}\left(v_{*}+w\right) \Delta w+\Psi^{\prime}\left(v_{*}+w\right)^{-1} D \Psi^{\prime \prime}\left(v_{*}+w\right)[\nabla w, \nabla w]+f_{\mathrm{nf}}^{0}\left(v_{*}+w\right)$, where now $f_{\mathrm{nf}}^{0}(v)=(0, g(v))^{\top}$. In what follows, the first component of the vector $w$ will play a distinguished role, as is clear from the expression of $f_{\mathrm{nf}}^{0}$. Thus we shall often write $w=\left(w_{0}, w_{\mathrm{h}}\right)^{\top}$, with $w_{0} \in \mathbb{R}$ and $w_{\mathrm{h}} \in \mathbb{R}^{N-1}$.

\section{LineAR EVOLUTION ESTIMATES}

We consider the linearization of (21) at $w=0$, which reads

$$
w_{t}=\Psi^{\prime}\left(v_{*}\right)^{-1} D \Psi^{\prime}\left(v_{*}\right) \Delta w+f_{\mathrm{nf}}^{\prime}\left(v_{*}\right) w .
$$

To simplify the notation, we define

$$
A(t)=\Psi^{\prime}\left(v_{*}(t)\right) \quad \text { and } \quad B(t)=\left(\begin{array}{cc}
0 & 0 \\
0 & L(\omega t)
\end{array}\right),
$$

where $L(\theta)$ is the $(N-1) \times(N-1)$ matrix which appears in (18). Note that $A(t), B(t)$ are $T$-periodic $N \times N$ matrices, and that $A(t)$ is invertible for all $t$. The linearization (22) then becomes

$$
w_{t}=A(t)^{-1} D A(t) \Delta w+B(t) w, \quad t \in \mathbb{R} .
$$

By Fourier duality this system is equivalent to the family of ODEs

$$
w_{t}=-k^{2} A(t)^{-1} D A(t) w+B(t) w, \quad t \in \mathbb{R}, \quad k \in \mathbb{R}^{n} .
$$

Since (24) is related to (3) by the $T$-periodic linear transformation $u=\Psi^{\prime}\left(v_{*}(t)\right) w$, it is clear that the Floquet spectrum of (24) is identical to that of (3) and therefore satisfies Hypothesis [1.2. Let $M(t, s ; k)$ denote the evolution operator defined by (24), so that any solution of (24) satisfies $w(t)=M(t, s ; k) w(s)$ for $t \geq s$. As $w=\left(w_{0}, w_{\mathrm{h}}\right)^{\top} \in \mathbb{R} \times \mathbb{R}^{N-1}$, it is natural to decompose the matrix $M$ in blocks as follows:

$$
M(t, s ; k)=\left(\begin{array}{ll}
M_{00}(t, s ; k) & M_{0 \mathrm{~h}}(t, s ; k) \\
M_{\mathrm{h} 0}(t, s ; k) & M_{\mathrm{hh}}(t, s ; k)
\end{array}\right),
$$


where $M_{00}, M_{0 \mathrm{~h}}, M_{\mathrm{h} 0}, M_{\mathrm{hh}}$ are matrices of size $1 \times 1,1 \times(N-1),(N-1) \times 1$, $(N-1) \times(N-1)$, respectively. The main result of this section is the following pointwise estimate on $M(t, s ; k)$ :

Proposition 4.1 (Pointwise estimates). There exist constants $C, d>0$ such that, for all $t \geq s$ and all $k \in \mathbb{R}^{n}$, one has

$$
\begin{aligned}
\left|M_{00}(t, s ; k)\right| & \leq C \mathrm{e}^{-d k^{2}(t-s)}, \\
\left|M_{0 \mathrm{~h}}(t, s ; k)\right|+\left|M_{\mathrm{h} 0}(t, s ; k)\right| & \leq \frac{C}{1+t-s} \mathrm{e}^{-d k^{2}(t-s)}, \\
\left|M_{\mathrm{hh}}(t, s ; k)\right| & \leq \frac{C}{(1+t-s)^{2}} \mathrm{e}^{-d k^{2}(t-s)},
\end{aligned}
$$

where the norms on the left-hand side are arbitrary, $k$-independent matrix norms.

Proof. Since the coefficients in (24) are $T$-periodic, we have $M(t+T, s+T ; k)=$ $M(t, s ; k)$ for all $t, s \in \mathbb{R}$ and all $k \in \mathbb{R}^{n}$. As a consequence, if $t \geq s$ and if $\tau_{1}, \tau_{2} \in[0, T)$ are such that $t-\tau_{1}$ and $s+\tau_{2}$ are integer multiples of $T$, we have the identity

$$
M(t, s ; k)=M\left(t, t-\tau_{1} ; k\right) M(k)^{m} M\left(s+\tau_{2}, s ; k\right), \quad k \in \mathbb{R}^{n},
$$

where $M(k)=M(T, 0 ; k)$ and $m \in \mathbb{N}$ is such that $t-s=\tau_{1}+m T+\tau_{2}$. To prove Proposition 4.1, it is therefore sufficient to estimate $M(k)^{m}$ and $M(t, s ; k)$ for $0 \leq t-s \leq T$.

Step 1: Estimates on $M(k)^{m}$.

The general strategy is to distinguish between various parameter regimes. For small and intermediate values of $k$, we essentially exploit Hypothesis 1.2, while for large $k$ it is sufficient to use the parabolicity of (24).

Small k: We solve the ODE (24) perturbatively for $t \in[0, T]$ and obtain

$$
w(t)=U(t, 0) w(0)-k^{2} \int_{0}^{t} U(t, s) A(s)^{-1} D A(s) U(s, 0) w(0) \mathrm{d} s+\mathrm{O}\left(k^{4}\right),
$$

where $U(t, s)=M(t, s ; 0)$ is the evolution operator associated to the equation $w_{t}=B(t) w$. In particular, setting $t=T$, we find

$$
\begin{aligned}
M(k) & =U(T, 0)\left(\mathrm{id}-k^{2} \int_{0}^{T}(A(t) U(t, 0))^{-1} D(A(t) U(t, 0)) \mathrm{d} t+\mathrm{O}\left(k^{4}\right)\right) \\
& =\left(\begin{array}{cc}
1 & 0 \\
0 & V
\end{array}\right)\left(\begin{array}{cc}
1-d_{0} T k^{2}+\mathrm{O}\left(k^{4}\right) & \mathrm{O}\left(k^{2}\right) \\
\mathrm{O}\left(k^{2}\right) & \mathrm{id}+\mathrm{O}\left(k^{2}\right)
\end{array}\right),
\end{aligned}
$$

where $V$ is the $(N-1) \times(N-1)$ Floquet matrix associated to the $T$-periodic linear operator $L(\omega t)$, and

$$
d_{0}=\frac{1}{T} \int_{0}^{T} e_{1}^{\top}(A(t) U(t, 0))^{-1} D(A(t) U(t, 0)) e_{1} \mathrm{~d} t=\frac{1}{T} \int_{0}^{T} e_{1}^{\top} A(t)^{-1} D A(t) e_{1} \mathrm{~d} t .
$$

Here $e_{1}=(1,0)^{\top}$ is the first vector of the canonical basis in $\mathbb{R}^{N}$. As was already observed, all eigenvalues of $V$ are contained in the disk $\left\{z \in \mathbb{C}|| z \mid<e^{-\nu_{1} T}\right\}$ for some $\nu_{1}>0$, and it follows from (29) that $M(k)$ has exactly $N-1$ eigenvalues in that disk if $k$ is sufficiently small. The remaining Floquet multiplier has the expansion $1-d_{0} T k^{2}+\mathrm{O}\left(k^{4}\right)$, in agreement with Hypothesis [1.2. Incidentally, we observe that (30) is identical to (4). Indeed, since $u_{*}(t)=\Psi\left(v_{*}(t)\right)=\Psi\left(\omega t e_{1}\right)$, we 
have $u_{*}^{\prime}(t)=\omega \Psi^{\prime}\left(v_{*}(t)\right) e_{1}=\omega A(t) e_{1}$, and it is also straightforward to verify that the bounded solution of the adjoint equation (5) is $U_{*}(t)=\left(A(t)^{-1}\right)^{\top} e_{1}$. Thus (30) can be written as

$$
d_{0}=\frac{1}{\omega T} \int_{0}^{T} U_{*}(t)^{\top} D u_{*}^{\prime}(t) \mathrm{d} t=\frac{\int_{0}^{T} U_{*}(t)^{\top} D u_{*}^{\prime}(t) \mathrm{d} t}{\int_{0}^{T} U_{*}(t)^{\top} u_{*}^{\prime}(t) \mathrm{d} t},
$$

and Hypothesis 1.2 guarantees that $d_{0}>0$.

For $k \in \mathbb{R}^{n}$ sufficiently small, let $P(k)$ denote the spectral projection onto the one-dimensional eigenspace of $M(k)$ corresponding to the neutral Floquet exponent $\lambda_{1}(k)=-d_{0} k^{2}+\mathrm{O}\left(k^{4}\right)$. From (29) it is easy to verify that $P(k)$ has the following form:

$$
P(k)=\frac{1}{1+k^{4} b^{\top} a}\left(\begin{array}{cc}
1 & k^{2} b^{\top} \\
k^{2} a & k^{4} a b^{\top}
\end{array}\right),
$$

where $a(k), b(k)$ are $(N-1)$-dimensional vectors with $a(k), b(k)=\mathrm{O}(1)$ as $k \rightarrow 0$. By construction, we have for any $m \in \mathbb{N}$ :

$M(k)^{m}=M(k)^{m} P(k)+M(k)^{m}($ id $-P(k))=\mathrm{e}^{m T \lambda_{1}(k)} P(k)+(M(k)(\text { id }-P(k)))^{m}$.

Since $\lambda_{1}(k) \leq-d_{1} k^{2}$ for small $k$ if $0<d_{1}<d_{0}$, and since the spectral radius of $M(k)($ id $-P(k))$ is smaller than $e^{-\nu_{1} T}$, we conclude that

$$
\left|M(k)^{m}\right| \leq C \mathrm{e}^{-d_{1} k^{2} m T}\left(\begin{array}{cc}
1 & k^{2} \\
k^{2} & k^{4}
\end{array}\right)+C \mathrm{e}^{-\nu_{1} m T}\left(\begin{array}{cc}
k^{4} & k^{2} \\
k^{2} & 1
\end{array}\right)
$$

for all $m \in \mathbb{N}$ if $|k| \leq \kappa_{0} \ll 1$. Here the matrix norm $|\cdot|$ is applied separately to each of the four blocks of $M(k)^{m}$, and for convenience the four upper bounds are collected in $2 \times 2$ matrices.

Large $k$ : In this parameter regime, it is more convenient to set $v(t)=A(t) w(t)$ and to solve the $v$-equation corresponding to (24), namely

$$
v_{t}=-k^{2} D v+C(t) v, \quad \text { where } \quad C(t)=A^{\prime}(t) A(t)^{-1}+A(t) B(t) A(t)^{-1} .
$$

The matrix $C(t)$ is $T$-periodic, hence uniformly bounded. A standard energy estimate yields

$$
\frac{1}{2} \frac{\mathrm{d}}{\mathrm{d} t}\|v(t)\|_{2}^{2}=-k^{2}(v(t), D v(t))+(v(t), C(t) v(t)) \leq-d_{2} k^{2}\|v(t)\|_{2}^{2}+K\|v(t)\|_{2}^{2},
$$

where $d_{2}>0$ is the smallest eigenvalue of the (symmetric and positive) matrix $D$, and $K=\sup _{t \in[0, T]}\|C(t)\|_{2}$. Thus any solution of (32) satisfies $\|v(t)\|_{2} \leq$ $\mathrm{e}^{\left(-d_{2} k^{2}+K\right) t}\|v(0)\|_{2}$, and returning to the $w$-equation we obtain

$$
\|w(t)\|_{2} \leq C \mathrm{e}^{\left(-d_{2} k^{2}+K\right) t}\|w(0)\|_{2}, \quad t \geq 0,
$$

for some $C>0$. In particular, if we choose $t=m T$ and if we assume that $|k| \geq \kappa_{1}$ with $\kappa_{1}=\left(2 K / d_{2}\right)^{1 / 2}$, we arrive at

$$
\left|M(k)^{m}\right| \leq C \mathrm{e}^{-d_{3} k^{2} m T}, \quad \text { where } \quad d_{3}=\frac{d_{2}}{2 T} .
$$


Intermediate $k$ : By Hypothesis [1.2, if $\kappa_{0} \leq|k| \leq \kappa_{1}$, the spectrum of $M(k)$ is entirely contained in the disk $\left\{z \in \mathbb{C}|| z \mid<\mathrm{e}^{-\mu T}\right\}$ for some $\mu>0$. Moreover, the resolvent matrix $(z-M(k))^{-1}$ is uniformly bounded for all $z$ on the circle $\left\{|z|=\mathrm{e}^{-\mu T}\right\}$ and all $k$ in the annulus $\kappa_{0} \leq|k| \leq \kappa_{1}$. If $0<d_{4}<\mu / \kappa_{1}^{2}$, it follows that

$$
\left|M(k)^{m}\right| \leq C \mathrm{e}^{-d_{4} k^{2} m T}, \quad m \in \mathbb{N},
$$

where the constant $C$ is independent of $k$.

Summarizing the results obtained so far, we have proved that there exist $C>0$, $d>0$, and $\nu>0$ such that

$$
\left|M(k)^{m}\right| \leq C \mathrm{e}^{-d k^{2} m T}\left(\begin{array}{cc}
1 & \hat{k}^{2} \\
\hat{k}^{2} & \hat{k}^{4}+\mathrm{e}^{-\nu m T}
\end{array}\right)
$$

for all $k \in \mathbb{R}^{n}$ and all $m \in \mathbb{N}$, where $\hat{k}^{2}=\min \left(k^{2}, 1\right)$.

Step 2: Estimates on $M(t, s ; k)$ for $0 \leq t-s \leq T$.

Our goal is to show that

$$
|M(t, s ; k)| \leq C \mathrm{e}^{-d k^{2}(t-s)}\left(\begin{array}{cc}
1 & \min \left\{k^{2}(t-s), 1\right\} \\
\min \left\{k^{2}(t-s), 1\right\} & 1
\end{array}\right),
$$

for some $C>0$ and $d>0$. In the case where $k^{2}(t-s)$ is small, say $k^{2}(t-s) \leq$ $\kappa_{2} \ll 1$, we can solve (24) perturbatively and obtain as in (29),

$$
M(t, s ; k)=\left(\begin{array}{cc}
1 & 0 \\
0 & V(t, s)
\end{array}\right)\left(\mathrm{id}+\mathrm{O}\left(k^{2}(t-s)\right)\right),
$$

from which (35) follows (for any fixed $d>0$ ). If $k^{2}(t-s) \geq \kappa_{2}$, then using (33) we immediately find $|M(t, s ; k)| \leq C \mathrm{e}^{\left(-d_{2} k^{2}+K\right)(t-s)} \leq C \mathrm{e}^{K T} \mathrm{e}^{-d_{2} k^{2}(t-s)}$, and this implies (35) with $d=d_{2}$.

It is now easy to conclude the proof of Proposition 4.1. We claim that there exist $C>0, d>0$, and $\nu>0$ such that

$$
|M(t, s ; k)| \leq C \mathrm{e}^{-d k^{2}(t-s)}\left(\begin{array}{cc}
1 & \hat{k}^{2} \\
\hat{k}^{2} & \hat{k}^{4}+\mathrm{e}^{-\nu(t-s)}
\end{array}\right),
$$

for all $k \in \mathbb{R}^{n}$ and all $t \geq s$, where $\hat{k}^{2}=\min \left(k^{2}, 1\right)$. Estimate (37) reduces to (34) when $t-s=m T$, and follows from (35) when $t-s \leq T$. In the case where $t-s>T$, we decompose $t-s=\tau_{1}+m T+\tau_{2}$ with $\tau_{1}, \tau_{2} \in[0, T)$ and $m \in \mathbb{N}$, and we factorize $M(t, s ; k)$ as in (28). Using (34), (35), we find

$$
|M(t, s ; k)| \leq C \mathrm{e}^{-d k^{2}(t-s)}\left(\begin{array}{cc}
1 & \hat{k}^{2} \\
\hat{k}^{2} & 1
\end{array}\right)\left(\begin{array}{cc}
1 & \hat{k}^{2} \\
\hat{k}^{2} & \hat{k}^{4}+\mathrm{e}^{-\nu m T}
\end{array}\right)\left(\begin{array}{cc}
1 & \hat{k}^{2} \\
\hat{k}^{2} & 1
\end{array}\right),
$$

and (37) easily follows. Finally, it is straightforward to verify that (37) implies (25), (26), (27), possibly with a slightly smaller value of $d>0$. This concludes the proof.

Remark 4.2. For all $k \in \mathbb{R}^{n}$ and all $s \in \mathbb{R}$, we have

$$
\lim _{t \rightarrow+\infty} M\left(t, s ; k t^{-1 / 2}\right)=\mathrm{e}^{-d_{0} k^{2}}\left(\begin{array}{ll}
1 & 0 \\
0 & 0
\end{array}\right),
$$

where $d_{0}$ is as in Hypothesis 1.2. This follows from the proof of Proposition 4.1, and in particular from (28), (31), (36). 
Proposition 4.3 (Estimates for the derivatives). For any $\alpha \in \mathbb{N}^{n}$ there exists $C>0$ such that, for all $t>s$ and all $k \in \mathbb{R}^{n}$,

$$
\left|\partial_{k}^{\alpha} M(t, s ; k)\right| \leq C(t-s)^{|\alpha| / 2} \mathrm{e}^{-d k^{2}(t-s)} .
$$

The same estimate holds for $M_{00},(1+t-s) M_{0 \mathrm{~h}},(1+t-s) M_{\mathrm{h} 0}$, and $(1+t-s)^{2} M_{\mathrm{hh}}$.

Proof. It is clear from (24) that $M(t, s ; k)$ depends on the parameter $k \in \mathbb{R}^{n}$ only through the scalar quantity $p=k^{2}$. In this proof, we set $M(t, s ; k)=\tilde{M}\left(t, s ; k^{2}\right)$ and we consider the derivatives of $\tilde{M}(t, s ; p)$ with respect to $p$. Our goal is to prove the estimate

$$
\left|\partial_{p}^{j} \tilde{M}(t, s ; p)\right| \leq C_{j}(t-s)^{j} \mathrm{e}^{-d p(t-s)}, \quad j \in \mathbb{N},
$$

which immediately implies (38). Differentiating (24) with respect to $p=k^{2}$, we find

$$
\left(\partial_{p} w\right)_{t}=-\mathcal{D}(t) w+(B(t)-p \mathcal{D}(t))\left(\partial_{p} w\right),
$$

where $\mathcal{D}(t)=A(t)^{-1} D A(t)$. Since $\partial_{p} \tilde{M}(s, s ; p)=0$, we deduce that

$$
\partial_{p} \tilde{M}(t, s ; p)=-\int_{s}^{t} \tilde{M}\left(t, t_{1} ; p\right) \mathcal{D}\left(t_{1}\right) \tilde{M}\left(t_{1}, s ; p\right) \mathrm{d} t_{1} .
$$

Iterating this procedure, we obtain for any $j \in \mathbb{N}$ the representation formula

$$
\begin{aligned}
\partial_{p}^{j} \tilde{M}(t, s ; p)= & (-1)^{j} j ! \int_{s}^{t} \int_{s}^{t_{1}} \ldots \int_{s}^{t_{j-1}} \tilde{M}\left(t, t_{1} ; p\right) \mathcal{D}\left(t_{1}\right) \\
& \times M\left(t_{1}, t_{2} ; p\right) \mathcal{D}\left(t_{2}\right) \ldots M\left(t_{j-1}, t_{j} ; p\right) \mathcal{D}\left(t_{j}\right) M\left(t_{j}, s ; p\right) \mathrm{d} t_{j} \ldots \mathrm{d} t_{1} .
\end{aligned}
$$

Now, if we use the pointwise estimate $|\tilde{M}(t, s ; p)| \leq C \mathrm{e}^{-d p(t-s)}$ established in Proposition 4.1, we easily obtain (39). To improve this bound and obtain faster decay rates in time for the submatrices $\tilde{M}_{0 \mathrm{~h}}, \tilde{M}_{\mathrm{h} 0}$, and $\tilde{M}_{\mathrm{hh}}$, we use the more precise estimates on $\tilde{M}(t, s ; p)$ which follow from (37). The calculations are straightforward and can be left to the reader.

We now convert our estimates on the Fourier multipliers $M(t, s ; k)$ into bounds on the linear evolution equation (23) in various $L^{p}$ spaces. The two-parameter semigroup $\mathcal{M}(t, s)$ associated to (23) is defined using the Fourier transform by the relation

$$
(\widehat{\mathcal{M}(t, s)} v)(k)=M(t, s ; k) \hat{v}(k), \quad k \in \mathbb{R}^{n} .
$$

The following proposition contains the main estimates on $\mathcal{M}(t, s)$ which will be used in the nonlinear stability proof.

Proposition 4.4 ( $L^{p}-L^{q}$ estimates). There exists a positive constant $C$ such that, for all $t>s$ and all $1 \leq p \leq q \leq \infty$, one has

$$
\|\mathcal{M}(t, s) v\|_{L^{q}\left(\mathbb{R}^{n}\right)} \leq \frac{C}{(t-s)^{\frac{n}{2}\left(\frac{1}{p}-\frac{1}{q}\right)}}\|v\|_{L^{p}\left(\mathbb{R}^{n}\right)} .
$$

The same estimate holds for $\mathcal{M}_{00},(1+t-s) \mathcal{M}_{0 \mathrm{~h}},(1+t-s) \mathcal{M}_{\mathrm{h} 0}$, and $(1+t-s)^{2} \mathcal{M}_{\mathrm{hh}}$.

Proof. By construction $\mathcal{M}(t, s)$ is the convolution operator with the function $x \mapsto$ $\mathcal{M}(t, s ; x)$, which is just the inverse Fourier transform of $k \mapsto M(t, s ; k)$. Thus using the pointwise estimate (25), we easily obtain

$$
\|\mathcal{M}(t, s ; \cdot)\|_{L^{\infty}\left(\mathbb{R}^{n}\right)} \leq C\|M(t, s ; \cdot)\|_{L^{1}\left(\mathbb{R}^{n}\right)} \leq \frac{C}{(t-s)^{n / 2}} .
$$


To estimate the $L^{1}$ norm of $\mathcal{M}(t, s ; \cdot)$, we use Sobolev embeddings. Let $m \in \mathbb{N}$ be the smallest integer such that $m>n / 2$. Using the estimates of Proposition 4.3 together with Hölder's inequality and Parseval's identity, we find

$$
\begin{gathered}
\|\mathcal{M}(t, s ; \cdot)\|_{L^{1}\left(\mathbb{R}^{n}\right)}=\int_{\mathbb{R}^{n}}\left(1+\frac{|x|^{2}}{t-s}\right)^{-m / 2}\left(1+\frac{|x|^{2}}{t-s}\right)^{m / 2}|\mathcal{M}(t, s ; x)| \mathrm{d} x \\
\leq C(t-s)^{n / 4}\left(\int_{\mathbb{R}^{n}}\left(1+\frac{|x|^{2}}{t-s}\right)^{m}|\mathcal{M}(t, s ; x)|^{2} \mathrm{~d} x\right)^{1 / 2} \\
\leq C(t-s)^{n / 4}\left(\int_{\mathbb{R}^{n}} \sum_{|\alpha| \leq m}(t-s)^{-|\alpha|}\left|\partial_{k}^{\alpha} M(t, s ; k)\right|^{2} \mathrm{~d} k\right)^{1 / 2} \leq C .
\end{gathered}
$$

Summarizing, we have shown that

$$
\|\mathcal{M}(t, s ; \cdot)\|_{L^{p}\left(\mathbb{R}^{n}\right)} \leq \frac{C}{(t-s)^{\frac{n}{2}\left(1-\frac{1}{p}\right)}}, \quad 1 \leq p \leq \infty,
$$

and (41) follows by Young's inequality. The estimates for $\mathcal{M}_{00},(1+t-s) \mathcal{M}_{0 \mathrm{~h}}$, $(1+t-s) \mathcal{M}_{\mathrm{h} 0}$, and $(1+t-s)^{2} \mathcal{M}_{\mathrm{hh}}$ are proved in exactly the same way.

Remark 4.5. Under the assumptions of Proposition 4.4, we also have

$$
\left\|\partial_{x}^{\alpha} \mathcal{M}(t, s) v\right\|_{L^{q}\left(\mathbb{R}^{n}\right)} \leq \frac{C_{\alpha}}{(t-s)^{\frac{n}{2}\left(\frac{1}{p}-\frac{1}{q}\right)+\frac{|\alpha|}{2}}}\|v\|_{L^{p}\left(\mathbb{R}^{n}\right)}, \quad \alpha \in \mathbb{N}^{n},
$$

and the same estimates hold for $\mathcal{M}_{00},(1+t-s) \mathcal{M}_{0 \mathrm{~h}},(1+t-s) \mathcal{M}_{\mathrm{h} 0}$, and $(1+t-$ $s)^{2} \mathcal{M}_{\mathrm{hh}}$. This is obvious in view of Proposition 4.1, since the operator $\partial_{x}^{\alpha} \mathcal{M}(t, s)$ is the Fourier multiplier associated to the function $(i k)^{\alpha} M(t, s ; k)$.

Remark 4.6. For later use, we also observe that, if $t>0$ and $0<s<t$, then

$$
\left\|\left(\mathcal{M}_{00}(t, s)-\mathcal{M}_{00}(t, 0)\right) v\right\|_{L^{q}\left(\mathbb{R}^{n}\right)} \leq \frac{C}{(t-s)^{\frac{n}{2}\left(\frac{1}{p}-\frac{1}{q}\right)}} \frac{s}{t}\|v\|_{L^{p}\left(\mathbb{R}^{n}\right)},
$$

for $1 \leq p \leq q \leq \infty$. If $t / 2 \leq s \leq t$, this bound follows immediately from (41) and the triangle inequality. If $0<s \leq t / 2$, we observe that

$$
M_{00}(s, 0 ; k)=1+k^{2} s R_{0}(s, k), \quad M_{\mathrm{h} 0}(s, 0 ; k)=k^{2} s R_{\mathrm{h}}(s, k),
$$

where $R_{0}(s, k), R_{\mathrm{h}}(s, k)$ are uniformly bounded for $s>0$ and $k \in \mathbb{R}^{n}$; see (36). Since

$M_{00}(t, s ; k)-M_{00}(t, 0 ; k)=M_{00}(t, s ; k)\left(1-M_{00}(s, 0 ; k)\right)-M_{0 \mathrm{~h}}(t, s ; k) M_{\mathrm{h} 0}(s, 0 ; k)$, we obtain the pointwise bound

$$
\left|M_{00}(t, s ; k)-M_{00}(t, 0 ; k)\right| \leq C k^{2} s \mathrm{e}^{-k^{2} d(t-s)} \leq C \frac{s}{t-s} \mathrm{e}^{-k^{2} d^{\prime}(t-s)},
$$

for some $d^{\prime}<d$, which allows us to establish the $L^{p}-L^{q}$ estimate (42) using the same arguments as in the proof of Proposition 4.4,

Finally, to control the quasilinear terms in the perturbation equation we will use the maximal regularity properties of the evolution semigroup $\mathcal{M}(t, s)$. 
Proposition 4.7 (Maximal regularity of type $L^{r}$ ). For any $r \in(1,+\infty)$ and any $\tilde{T}>0$, there exists $C>0$ such that the following holds. If $v \in L^{r}\left((0, \tilde{T}), L^{2}\left(\mathbb{R}^{n}\right)\right)$ and if $w$ satisfies

$$
w(t)=\int_{0}^{t} \mathcal{M}(t, s) v(s) \mathrm{d} s, \quad t \in[0, \tilde{T}]
$$

then

$$
\int_{0}^{\tilde{T}}\|\Delta w(t)\|_{L^{2}}^{r} \mathrm{~d} t \leq C \int_{0}^{\tilde{T}}\|v(t)\|_{L^{2}}^{r} \mathrm{~d} t
$$

Proof. For any $t \in \mathbb{R}$ the generator $\mathcal{L}(t)=A(t)^{-1} D A(t) \Delta+B(t)$ is an elliptic operator in $L^{2}\left(\mathbb{R}^{n}\right)$ with (time-independent) domain $H^{2}\left(\mathbb{R}^{n}\right)$. Moreover $\mathcal{L}(t)$, considered as a bounded operator from $H^{2}\left(\mathbb{R}^{n}\right)$ into $L^{2}\left(\mathbb{R}^{n}\right)$, is a smooth function of $t$. Thus estimate (44) is a particular case of the results established in [16, 21].

We also state a corollary which will be useful in the next section.

Corollary 4.8. Fix $r \in(1, \infty)$ and $\alpha \in \mathbb{R}$. There exists $C>0$ such that, if

$$
w(t)=\int_{t-T}^{t} \mathcal{M}(t, s) v(s) \mathrm{d} s, \quad t \geq T,
$$

then

$$
\int_{T}^{\infty}(1+t)^{\alpha}\|\Delta w(t)\|_{L^{2}}^{r} \mathrm{~d} t \leq C \int_{0}^{\infty}(1+t)^{\alpha}\|v(t)\|_{L^{2}}^{r} \mathrm{~d} t
$$

Proof. Fix $k \in \mathbb{N}^{*}:=\mathbb{N} \backslash\{0\}$. If $t \in[k T,(k+1) T]$, where $T>0$ is the period of $\mathcal{L}(t)$, we can write

$$
w(t)=\int_{(k-1) T}^{t} \mathcal{M}(t, s) v(s) \mathrm{d} s-\int_{(k-1) T}^{t-T} \mathcal{M}(t, s) v(s) \mathrm{d} s .
$$

Since $\mathcal{M}(t+T, s+T)=\mathcal{M}(t, s)$, and since $0 \leq t-(k-1) T \leq 2 T$, we can use Proposition 4.7 (with $\tilde{T}=2 T$ ) to control both terms in the right-hand side. We obtain

$$
\int_{k T}^{(k+1) T}\|\Delta w(t)\|_{L^{2}}^{r} \mathrm{~d} t \leq C \int_{(k-1) T}^{(k+1) T}\|v(t)\|_{L^{2}}^{r} \mathrm{~d} t,
$$

where the constant $C>0$ is independent of $k$, due to the periodicity properties of $\mathcal{M}(t, s)$. Multiplying both sides by $(k T)^{\alpha} \approx(1+t)^{\alpha}$ and summing over $k \in \mathbb{N}^{*}$, we obtain the desired result.

\section{NONLINEAR STABILITY IN LOW Dimensions}

This section is devoted to the proof of Theorem 1.3 in the case $n \leq 3$. Smoothness of the change of coordinates $\Psi$ implies that it is sufficient to prove the decay estimate (6) for the transformed equation (21), with smallness assumptions on the initial data $w^{0}$. To simplify the notation, we rewrite (21) in the compact form

$$
w_{t}=\mathcal{L}(t) w+F(t, w, \Delta w)+G(t, w, \nabla w)+H(t, w),
$$


where $\mathcal{L}(t)=A(t)^{-1} D A(t) \Delta+B(t)$ is the linear operator studied in Section 4 and

$$
\begin{aligned}
F(t, w, \Delta w)= & \left(\Psi^{\prime}\left(v_{*}(t)+w\right)^{-1}-\Psi^{\prime}\left(v_{*}(t)\right)^{-1}\right) D\left(\Psi^{\prime}\left(v_{*}(t)+w\right)\right) \Delta w \\
& +\Psi^{\prime}\left(v_{*}(t)\right)^{-1} D\left(\Psi^{\prime}\left(v_{*}(t)+w\right)-\Psi^{\prime}\left(v_{*}(t)\right) \Delta w\right. \\
G(t, w, \nabla w)= & \Psi^{\prime}\left(v_{*}(t)+w\right)^{-1} D \Psi^{\prime \prime}\left(v_{*}(t)+w\right)[\nabla w, \nabla w] \\
H(t, w)= & \left(0, \hat{g}_{2}(t, w)\right)^{\top} \\
\hat{g}_{2}(t, w)= & \left(L\left(\omega t+w_{0}\right)-L(\omega t)\right) w_{\mathrm{h}}+g_{2}\left(v_{*}(t)+w\right)\left[w_{\mathrm{h}}, w_{\mathrm{h}}\right] .
\end{aligned}
$$

Clearly, $F(t, w, \Delta w)=\mathrm{O}(w \Delta w)$ and $G(t, w, \nabla w)=\mathrm{O}\left(|\nabla w|^{2}\right)$. More precisely, if $\|w\|_{L^{\infty}}$ is sufficiently small, we have

$$
\begin{array}{ll}
\|F(t, w, \Delta w)\|_{L^{1}} \leq C\|w\|_{L^{2}}\|\Delta w\|_{L^{2}}, & \|G(t, w, \nabla w)\|_{L^{1}} \leq C\|\nabla w\|_{L^{2}}^{2}, \\
\|F(t, w, \Delta w)\|_{L^{2}} \leq C\|w\|_{L^{\infty}}\|\Delta w\|_{L^{2}}, & \|G(t, w, \nabla w)\|_{L^{2}} \leq C\|\nabla w\|_{L^{4}}^{2} .
\end{array}
$$

Since

$$
\|\nabla w\|_{L^{2}}^{2} \leq C\|w\|_{L^{2}}\|\Delta w\|_{L^{2}} \quad \text { and } \quad\|\nabla w\|_{L^{4}}^{2} \leq C\|w\|_{L^{\infty}}\|\Delta w\|_{L^{2}},
$$

we find

$$
\begin{aligned}
\|F(t, w, \Delta w)\|_{L^{1}}+\|G(t, w, \nabla w)\|_{L^{1}} & \leq C\|w\|_{L^{2}}\|\Delta w\|_{L^{2}}, \\
\|F(t, w, \Delta w)\|_{L^{2}}+\|G(t, w, \nabla w)\|_{L^{2}} & \leq C\|w\|_{L^{\infty}}\|\Delta w\|_{L^{2}} .
\end{aligned}
$$

Under the same assumptions, we also have

$$
\|H(t, w)\|_{L^{1}} \leq C\|w\|_{L^{2}}\left\|w_{\mathrm{h}}\right\|_{L^{2}} \quad \text { and } \quad\|H(t, w)\|_{L^{2}} \leq C\|w\|_{L^{2}}\left\|w_{\mathrm{h}}\right\|_{L^{\infty}} .
$$

Setting $K(t, w, \nabla w, \Delta w)=F(t, w, \Delta w)+G(t, w, \nabla w)$, we can write the integral equation associated with (21) in the form

(49) $w(t)=\mathcal{M}(t, 0) w^{0}+\int_{0}^{t} \mathcal{M}(t, s) K(s, w, \nabla w, \Delta w) \mathrm{d} s+\int_{0}^{t} \mathcal{M}(t, s) H(s, w) \mathrm{d} s$.

We now describe the function space in which we shall look for solutions of (49). Assume that $n \leq 3$ and choose $r \in(4,+\infty)$ so that

$$
\frac{1}{r}<\frac{n}{4}<1-\frac{1}{r} \text {. }
$$

We define the Banach space

$Y=\left\{w \in C^{0}\left([0,+\infty), L^{1}\left(\mathbb{R}^{n}\right) \cap L^{\infty}\left(\mathbb{R}^{n}\right)\right) \cap L^{r}\left((0,+\infty), \dot{H}^{2}\left(\mathbb{R}^{n}\right)\right) \mid\|w\|_{Y}<\infty\right\}$, where

$$
\|w\|_{Y}=\sup _{t \geq 0}\|w(t)\|_{L^{1}}+\sup _{t \geq 0}(1+t)^{n / 2}\|w(t)\|_{L^{\infty}}+\left(\int_{0}^{\infty}(1+t)^{r}\|\Delta w(t)\|_{L^{2}}^{r} \mathrm{~d} t\right)^{1 / r} .
$$

Lemma 5.1. If $w^{0} \in L^{1}\left(\mathbb{R}^{n}\right) \cap H^{2}\left(\mathbb{R}^{n}\right)$, the linear solution $W_{0}(t)=\mathcal{M}(t, 0) w^{0}$ belongs to $Y$ and $\left\|W_{0}\right\|_{Y} \leq C_{1}\left(\left\|w^{0}\right\|_{L^{1}}+\left\|w^{0}\right\|_{H^{2}}\right)$ for some $C_{1}>0$.

Proof. Since $n \leq 3$, we have $H^{2}\left(\mathbb{R}^{n}\right) \hookrightarrow L^{\infty}\left(\mathbb{R}^{n}\right)$; hence $w^{0} \in L^{1}\left(\mathbb{R}^{n}\right) \cap L^{\infty}\left(\mathbb{R}^{n}\right)$. Thus, using the linear estimates established in Proposition 4.4 we obtain

$$
\sup _{t \geq 0}\left\|W_{0}(t)\right\|_{L^{1}}+\sup _{t \geq 0}(1+t)^{n / 2}\left\|W_{0}(t)\right\|_{L^{\infty}} \leq C\left(\left\|w^{0}\right\|_{L^{1}}+\left\|w^{0}\right\|_{L^{\infty}}\right) .
$$


On the other hand, using Proposition 4.4 and Remark 4.5, we find

$$
\left\|\Delta W_{0}(t)\right\|_{L^{2}} \leq C\left\|w^{0}\right\|_{H^{2}} \quad \text { for } t \leq 1, \quad\left\|\Delta W_{0}(t)\right\|_{L^{2}} \leq \frac{C\left\|w^{0}\right\|_{L^{1}}}{t^{1+n / 4}} \text { for } t \geq 1 .
$$

As $r n / 4>1$ by (50), we conclude that

$$
\left(\int_{0}^{\infty}(1+t)^{r}\left\|\Delta W_{0}(t)\right\|_{L^{2}}^{r} \mathrm{~d} t\right)^{1 / r} \leq C\left(\left\|w^{0}\right\|_{L^{1}}+\left\|w^{0}\right\|_{H^{2}}\right)
$$

The next step consists in estimating the integral terms in (49), namely

$$
\mathcal{I}(t)=\int_{0}^{t} \mathcal{M}(t, s) K(s, w, \nabla w, \Delta w) \mathrm{d} s \quad \text { and } \quad \mathcal{J}(t)=\int_{0}^{t} \mathcal{M}(t, s) H(s, w) \mathrm{d} s .
$$

Proposition 5.2. There exist $C_{2}>0$ and $\delta_{2}>0$ such that, for all $w \in Y$ with $\|w\|_{Y} \leq \delta_{2}$, we have

$$
\|\mathcal{I}\|_{Y}+\|\mathcal{J}\|_{Y} \leq C_{2}\|w\|_{Y}^{2}
$$

Proof. Throughout the proof $C$ denotes a constant that changes between estimates, but does not depend on $w$. The smallness of $w$ in $Y$ implies that estimates (47) and (48) hold for the nonlinearities.

Estimate on $\|\mathcal{I}(t)\|_{L^{1}}$

Using (41) with $p=q=1$ and the first estimate in (47), we find

$\|\mathcal{I}(t)\|_{L^{1}} \leq C \int_{0}^{t}\|w(s)\|_{L^{2}}\|\Delta w(s)\|_{L^{2}} \mathrm{~d} s$

$$
\begin{aligned}
& \leq C\|w\|_{Y} \int_{0}^{t} \frac{1}{(1+s)^{\frac{n}{4}+1}}(1+s)\|\Delta w(s)\|_{L^{2}} \mathrm{~d} s \\
& \leq C\|w\|_{Y}\left(\int_{0}^{t} \frac{1}{(1+s)^{\left(\frac{n}{4}+1\right) \frac{r}{r-1}}} \mathrm{~d} s\right)^{1-\frac{1}{r}}\left(\int_{0}^{t}(1+s)^{r}\|\Delta w(s)\|_{L^{2}}^{r} \mathrm{~d} s\right)^{1 / r} .
\end{aligned}
$$

In the second inequality we used the bound $\|w(s)\|_{L^{2}} \leq\|w(s)\|_{L^{1}}^{1 / 2}\|w(s)\|_{L^{\infty}}^{1 / 2} \leq$ $\|w\|_{Y}(1+s)^{-n / 4}$, and in the last line we used Hölder's inequality. Taking the supremum over $t \geq 0$ and using (51), we conclude that

$$
\sup _{t \geq 0}\|\mathcal{I}(t)\|_{L^{1}} \leq C\|w\|_{Y}^{2} .
$$

Estimate on $\|\mathcal{I}(t)\|_{L^{\infty}}$

For $t \leq 1$, we use (41) with $(p, q)=(2, \infty)$ and the second estimate in (47). We obtain

$$
\|\mathcal{I}(t)\|_{L^{\infty}} \leq C \int_{0}^{t} \frac{1}{(t-s)^{n / 4}}\|w(s)\|_{L^{\infty}}\|\Delta w(s)\|_{L^{2}} \mathrm{~d} s \leq C t^{1-\frac{n}{4}-\frac{1}{r}}\|w\|_{Y}^{2},
$$

where the last estimate is again a consequence of Hölder's inequality. Note that $1-\frac{n}{4}-\frac{1}{r}>0$ by (50). For $t \geq 1$ we split

$$
\begin{aligned}
\mathcal{I}(t) & =\int_{0}^{t / 2} \mathcal{M}(t, s) K(s, w, \nabla w, \Delta w) \mathrm{d} s+\int_{t / 2}^{t} \mathcal{M}(t, s) K(s, w, \nabla w, \Delta w) \mathrm{d} s \\
& =: \mathcal{I}_{1}(t)+\mathcal{I}_{2}(t) .
\end{aligned}
$$


Using (41) with $(p, q)=(1, \infty)$ and proceeding as in (52), we find

$$
\begin{aligned}
(1+t)^{n / 2}\left\|\mathcal{I}_{1}(t)\right\|_{L^{\infty}} & \leq C(1+t)^{n / 2} \int_{0}^{t / 2} \frac{1}{(t-s)^{n / 2}}\|w(s)\|_{L^{2}}\|\Delta w(s)\|_{L^{2}} \mathrm{~d} s \\
& \leq C \int_{0}^{t / 2}\|w(s)\|_{L^{2}}\|\Delta w(s)\|_{L^{2}} \mathrm{~d} s \leq C\|w\|_{Y}^{2}
\end{aligned}
$$

On the other hand, using (41) with $(p, q)=(2, \infty)$ we obtain

$$
\begin{aligned}
(1+t)^{n / 2}\left\|\mathcal{I}_{2}(t)\right\|_{L^{\infty}} & \leq C(1+t)^{n / 2} \int_{t / 2}^{t} \frac{1}{(t-s)^{n / 4}}\|w(s)\|_{L^{\infty}}\|\Delta w(s)\|_{L^{2}} \mathrm{~d} s \\
& \leq C\|w\|_{Y} \frac{1}{(1+t)} \int_{t / 2}^{t} \frac{1}{(t-s)^{n / 4}}(1+s)\|\Delta w(s)\|_{L^{2}} \mathrm{~d} s \\
& \leq C\|w\|_{Y}^{2} \frac{1}{(1+t)^{\frac{n}{4}+\frac{1}{r}}}
\end{aligned}
$$

where in the last line we used Hölder's inequality as in (53). This shows that

$$
\sup _{t \geq 0}(1+t)^{n / 2}\|\mathcal{I}(t)\|_{L^{\infty}} \leq C\|w\|_{Y}^{2} .
$$

Estimate on $\|\Delta \mathcal{I}(t)\|_{L^{2}}$

The estimates for the second derivative require maximal regularity, Proposition 4.7. We need to estimate $\int_{0}^{\infty}(1+t)^{r}\|\Delta \mathcal{I}(t)\|_{L^{2}}^{r} \mathrm{~d} t$. We therefore split the integral and first estimate

$$
\int_{0}^{T}(1+t)^{r}\|\Delta \mathcal{I}(t)\|_{L^{2}}^{r} \mathrm{~d} t \leq C \int_{0}^{T}\|w(t)\|_{L^{\infty}}^{r}\|\Delta w(t)\|_{L^{2}}^{r} \mathrm{~d} t \leq C\|w\|_{Y}^{2 r},
$$

where we used Proposition 4.7 and estimate (47) in the first inequality, and the definition of $\|w\|_{Y}$ in the second inequality.

We next derive estimates for $\Delta \mathcal{I}(t)$ at $t \geq T$. Here it is more convenient to decompose

$$
\begin{aligned}
\mathcal{I}(t) & =\int_{0}^{t-T} \mathcal{M}(t, s) K(s, w, \nabla w, \Delta w) \mathrm{d} s+\int_{t-T}^{t} \mathcal{M}(t, s) K(s, w, \nabla w, \Delta w) \mathrm{d} s \\
& =: \mathcal{I}_{3}(t)+\mathcal{I}_{4}(t) .
\end{aligned}
$$

Using (41) with $(p, q)=(1,2)$ and Remark 4.5] we control the first term as

$$
\begin{aligned}
(1+t) & \left\|\Delta \mathcal{I}_{3}(t)\right\|_{L^{2}} \leq C(1+t) \int_{0}^{t-T} \frac{1}{(t-s)^{1+\frac{n}{4}}}\|w(s)\|_{L^{2}}\|\Delta w(s)\|_{L^{2}} \mathrm{~d} s \\
& \leq C\|w\|_{Y}(1+t) \int_{0}^{t-T} \frac{1}{(t-s)^{1+\frac{n}{4}}} \frac{1}{(1+s)^{1+\frac{n}{4}}}(1+s)\|\Delta w(s)\|_{L^{2}} \mathrm{~d} s \\
& \leq\|w\|_{Y}^{2} \frac{1}{(1+t)^{\frac{n}{4}}},
\end{aligned}
$$

where in the last estimate we used Hölder's inequality together with the fact that (56)

$$
\left\{\int_{0}^{t}\left(\frac{1}{1+t-s} \frac{1}{(t-s)^{n / 4}} \frac{1}{(1+s)^{1+\frac{n}{4}}}\right)^{q} \mathrm{~d} s\right\}^{1 / q} \leq \frac{C}{(1+t)^{1+\frac{n}{4}}}, \quad \text { for } 1 \leq q<\frac{4}{n} .
$$


Integrating over time and recalling that $n r / 4>1$, we obtain the desired bound for $\mathcal{I}_{3}$ :

$$
\int_{T}^{\infty}(1+t)^{r}\left\|\Delta \mathcal{I}_{3}(t)\right\|_{L^{2}}^{r} \mathrm{~d} t \leq C\|w\|_{Y}^{2 r}
$$

The other term $\mathcal{I}_{4}$ is estimated directly using (47) and Corollary 4.8

$$
\int_{T}^{\infty}(1+t)^{r}\left\|\Delta \mathcal{I}_{4}(t)\right\|_{L^{2}}^{r} \mathrm{~d} t \leq C \int_{0}^{\infty}(1+t)^{r}\|w(t)\|_{L^{\infty}}^{r}\|\Delta w(t)\|_{L^{2}}^{r} \mathrm{~d} t \leq C\|w\|_{Y}^{2 r}
$$

Estimates on $\|\mathcal{J}(t)\|_{L^{1}}$ and $\|\mathcal{J}(t)\|_{L^{\infty}}$

In this term, the nonlinearity does not contain derivatives which would yield decay, but we can exploit the stronger decay of the linear operator $\mathcal{M}(t, s)$ when acting on $H(s, w)$. Indeed, since $H(s, w)=\left(0, \hat{g}_{2}(s, w)\right)^{\top}$, we have

$$
\mathcal{M}(t, s) H(s, w)=\left(\begin{array}{c}
\mathcal{M}_{0 \mathrm{~h}}(t, s) \hat{g}_{2}(s, w) \\
\mathcal{M}_{\mathrm{hh}}(t, s) \hat{g}_{2}(s, w)
\end{array}\right)=: \mathcal{M}_{\cdot \mathrm{h}}(t, s) \hat{g}_{2}(s, w),
$$

and it follows from Proposition 4.4 that

$$
\|\mathcal{M}(t, s) H(s, w)\|_{L^{q}\left(\mathbb{R}^{n}\right)} \leq C \frac{1}{1+t-s} \frac{1}{(t-s)^{\frac{n}{2}\left(\frac{1}{p}-\frac{1}{q}\right)}}\|H(s, w)\|_{L^{p}\left(\mathbb{R}^{n}\right)},
$$

for $1 \leq p \leq q \leq \infty$. Using (57) with $p=q=1$ and estimate (48), we thus find

$$
\begin{aligned}
\|\mathcal{J}(t)\|_{L^{1}} & \leq C \int_{0}^{t} \frac{1}{1+t-s}\|w(s)\|_{L^{2}}^{2} \mathrm{~d} s \\
& \leq C\|w\|_{Y}^{2} \int_{0}^{t} \frac{1}{1+t-s} \frac{1}{(1+s)^{n / 2}} \mathrm{~d} s \leq C\|w\|_{Y}^{2} .
\end{aligned}
$$

In a similar way, using (57) with $(p, q)=(2, \infty)$, we arrive at

$$
\begin{aligned}
(1+t)^{n / 2}\|\mathcal{J}(t)\|_{L^{\infty}} & \leq C(1+t)^{n / 2} \int_{0}^{t} \frac{1}{1+t-s} \frac{1}{(t-s)^{n / 4}}\|w(s)\|_{L^{\infty}}\|w(s)\|_{L^{2}} \mathrm{~d} s \\
& \leq C\|w\|_{Y}^{2}(1+t)^{n / 2} \int_{0}^{t} \frac{1}{1+t-s} \frac{1}{(t-s)^{n / 4}} \frac{1}{(1+s)^{3 n / 4}} \mathrm{~d} s \\
& \leq C\|w\|_{Y}^{2} .
\end{aligned}
$$

Estimate on $\|\Delta \mathcal{J}(t)\|_{L^{2}}$

We first observe that

$$
\Delta \mathcal{J}(t)=\int_{0}^{t} \mathcal{M}_{\cdot \mathrm{h}}(t, s) \Delta \hat{g}_{2}(s, w) \mathrm{d} s .
$$

From the definition of $\hat{g}_{2}(t, w)$ (46), it is clear that $\left|\Delta \hat{g}_{2}(t, w)\right| \leq C\left(|w||\Delta w|+|\nabla w|^{2}\right)$, so that $\Delta \hat{g}_{2}$ satisfies the same estimates (47) as $F$ and $G$. Using the $L^{1}-L^{2}$ estimate for $\mathcal{M} \cdot \mathrm{h}(t, s)$, we thus find

$$
\begin{aligned}
(1+t) & \|\Delta \mathcal{J}(t)\|_{L^{2}} \leq C(1+t) \int_{0}^{t} \frac{1}{1+t-s} \frac{1}{(t-s)^{n / 4}}\|w(s)\|_{L^{2}}\|\Delta w(s)\|_{L^{2}} \mathrm{~d} s \\
& \leq C\|w\|_{Y}(1+t) \int_{0}^{t} \frac{1}{1+t-s} \frac{1}{(t-s)^{n / 4}} \frac{1}{(1+s)^{1+\frac{n}{4}}}(1+s)\|\Delta w(s)\|_{L^{2}} \mathrm{~d} s \\
& \leq C\|w\|_{Y}^{2} \frac{1}{(1+t)^{n / 4}}
\end{aligned}
$$


where in the last line we used Hölder's inequality and estimate (56). We deduce that

$$
\int_{0}^{\infty}(1+t)^{r}\|\Delta \mathcal{J}(t)\|_{L^{2}}^{r} \mathrm{~d} t \leq C\|w\|_{Y}^{2 r},
$$

and the proof of Proposition 5.2 is complete.

Proof of Theorem $1.3(n \leq 3)$. As was observed in Section 3, we can work with the transformed equation (21) instead of the original perturbation equation (12). Also, we can assume without loss of generality that the initial perturbation $w^{0}$ satisfies $\left\|w^{0}\right\|_{L^{1}}+\left\|w^{0}\right\|_{H^{2}} \leq \delta_{0}$ for some small $\delta_{0}>0$. Under these assumptions, we can solve equation (49) by a standard fixed point argument in the Banach space $Y$ defined by (51). Indeed, let $\mathcal{N}$ denote the right-hand side of (49), namely

$$
(\mathcal{N} w)(t)=\mathcal{M}(t, 0) w^{0}+\int_{0}^{t} \mathcal{M}(t, s) K(s, w, \nabla w, \Delta w) \mathrm{d} s+\int_{0}^{t} \mathcal{M}(t, s) H(s, w) \mathrm{d} s .
$$

If $w \in Y$ satisfies $\|w\|_{Y} \leq \delta_{2}$, where $\delta_{2}>0$ is as in Proposition 5.2, we know that $\mathcal{N} w \in Y$ and that

$$
\|\mathcal{N} w\|_{Y} \leq C_{1} \delta_{0}+C_{2}\|w\|_{Y}^{2} .
$$

Similar calculations show that

$$
\|\mathcal{N} w-\mathcal{N} \tilde{w}\|_{Y} \leq C_{2}\left(\|w\|_{Y}+\|\tilde{w}\|_{Y}\right)\|w-\tilde{w}\|_{Y}
$$

whenever $w, \tilde{w} \in Y$ with $\|w\|_{Y} \leq \delta_{2},\|\tilde{w}\|_{Y} \leq \delta_{2}$. Let $\mathcal{B} \subset Y$ denote the ball of radius $R=\min \left(2 C_{1} \delta_{0}, \delta_{2}\right)$ centered at the origin. If $\delta_{0}>0$ is small enough so that $2 C_{2} R<1$, it follows easily from (60), (61) that $\mathcal{N}(\mathcal{B}) \subset \mathcal{B}$ and that $\mathcal{N}$ is a strict contraction in $\mathcal{B}$. Let $w \in Y$ be the unique fixed point of $\mathcal{N}$ in $\mathcal{B}$. Then $w$ is a global solution of (21), and if we return to the original variables by setting $u(t, x)=\Psi\left(v_{*}(t)+w(t, x)\right)$, we obtain a global solution of (11) which satisfies the decay estimate (6) (with $t_{0}=0$ ), because

$$
\left|u(t, x)-u_{*}(t)\right|=\left|\Psi\left(v_{*}(t)+w(t, x)\right)-\Psi\left(v_{*}(t)\right)\right| \leq C \frac{\|w\|_{Y}}{(1+t)^{n / 2}}, \quad t \geq 0 .
$$

This concludes the proof.

Remark 5.3. The limitation $n \leq 3$ in the above proof is due to the fact that we use maximal regularity (MR) in the Hilbert space $L^{2}\left(\mathbb{R}^{n}\right)$ only; see e.g. (53). This choice was made for simplicity, but for equation (23) it is known that MR holds in all $L^{p}$ spaces with $1<p<\infty$; see [16, 21]. It is not difficult to verify that the argument above can be adapted to any space dimension $n$ if we use MR in $L^{p}\left(\mathbb{R}^{n}\right)$ with $p$ sufficiently large, depending on $n$.

\section{Asymptotic Behavior}

We know from Theorem 1.3 that small, localized perturbations of the periodic solution $u_{*}(t)$ converge to zero like $t^{-n / 2}$ as $t \rightarrow+\infty$. This decay rate is optimal in general, and it is even possible to compute the leading term in the asymptotic expansion of the perturbation as $t \rightarrow+\infty$. In this section, we assume (for simplicity) that $1 \leq n \leq 3$ and we consider the solution $w(t, x)$ of (21) with small initial data $w^{0} \in L^{1}\left(\mathbb{R}^{n}\right) \cap H^{2}\left(\mathbb{R}^{n}\right)$. If we decompose this solution as $w(t, x)=\left(w_{0}(t, x), w_{\mathrm{h}}(t, x)\right)^{\top}$, we first observe that the hyperbolic part $w_{\mathrm{h}}(t, x) \in \mathbb{R}^{N-1}$ has a faster decay as $t \rightarrow \infty$. 
Proposition 6.1. If the initial data $w^{0} \in L^{1}\left(\mathbb{R}^{n}\right) \cap H^{2}\left(\mathbb{R}^{n}\right)$ are sufficiently small, the hyperbolic component of the solution $w$ of (21) satisfies

$$
\sup _{t \geq 0}(1+t)\left\|w_{\mathrm{h}}(t)\right\|_{L^{1}}+\sup _{t \geq 0}(1+t)^{1+\frac{n}{2}}\left\|w_{\mathrm{h}}(t)\right\|_{L^{\infty}} \leq C\left(\left\|w^{0}\right\|_{L^{1}}+\left\|w^{0}\right\|_{H^{2}}\right) .
$$

Proof. Projecting the integral equation (49) onto the hyperbolic component, we find

$$
\begin{aligned}
w_{\mathrm{h}}(t) & =\mathcal{M}_{\mathrm{h} \cdot}(t, 0) w^{0}+\int_{0}^{t} \mathcal{M}_{\mathrm{h} \cdot}(t, s) K(s, w, \nabla w, \Delta w) \mathrm{d} s+\int_{0}^{t} \mathcal{M}_{\mathrm{h} \cdot}(t, s) H(s, w) \mathrm{d} s \\
& =: \mathcal{M}_{\mathrm{h} \cdot}(t, 0) w^{0}+\mathcal{I}_{\mathrm{h}}(t)+\mathcal{J}_{\mathrm{h}}(t),
\end{aligned}
$$

where $\mathcal{M}_{\mathrm{h} \cdot}(t, s)=\left(\mathcal{M}_{\mathrm{h} 0}(t, s), \mathcal{M}_{\mathrm{hh}}(t, s)\right)$. We know from Proposition 4.4 that

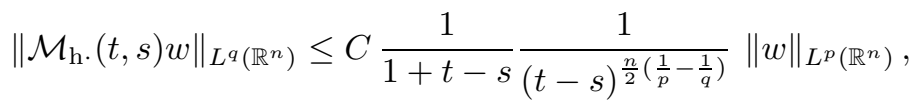

for $1 \leq p \leq q \leq \infty$. In particular, we have

$\sup _{t \geq 0}(1+t)\left\|\mathcal{M}_{\mathrm{h} \cdot}(t, 0) w^{0}\right\|_{L^{1}}+\sup _{t \geq 0}(1+t)^{1+\frac{n}{2}}\left\|\mathcal{M}_{\mathrm{h} \cdot}(t, 0) w^{0}\right\|_{L^{\infty}} \leq C\left(\left\|w^{0}\right\|_{L^{1}}+\left\|w^{0}\right\|_{L^{\infty}}\right)$.

Moreover, proceeding as in the proof of Proposition 5.2, we obtain

$$
\left\|\mathcal{I}_{\mathrm{h}}(t)\right\|_{L^{1}} \leq C \int_{0}^{t} \frac{1}{1+t-s} \frac{\|w\|_{Y}}{(1+s)^{1+\frac{n}{4}}}(1+s)\|\Delta w(s)\|_{L^{2}} \mathrm{~d} s \leq \frac{C}{1+t}\|w\|_{Y}^{2},
$$

and the same result holds for $(1+t)^{n / 2}\left\|\mathcal{I}_{\mathrm{h}}(t)\right\|_{L^{\infty}}$. Finally, to bound the term $\mathcal{J}_{\mathrm{h}}$, we observe that $\mathcal{M}_{\mathrm{h}} \cdot(t, s) H(s, w)=\mathcal{M}_{\mathrm{hh}}(t, s) \hat{g}_{2}(s, w)$ and we use the strong decay in time given by Proposition 4.4. We thus find

$$
\begin{aligned}
\left\|\mathcal{J}_{\mathrm{h}}(t)\right\|_{L^{1}} & \leq C \int_{0}^{t} \frac{1}{(1+t-s)^{2}}\|w(s)\|_{L^{1}}\left\|w_{\mathrm{h}}(s)\right\|_{L^{\infty}} \mathrm{d} s \\
& \leq C \int_{0}^{t} \frac{1}{(1+t-s)^{2}} \frac{\|w\|_{Y}^{2}}{(1+s)^{n / 2}} \mathrm{~d} s \leq \frac{C}{(1+t)^{n / 2}}\|w\|_{Y}^{2},
\end{aligned}
$$

and the same result holds for $(1+t)^{n / 2}\left\|\mathcal{J}_{\mathrm{h}}(t)\right\|_{L^{\infty}}$. This gives the desired result if $n \geq 2$. If $n=1$, we only have

$$
\sup _{t \geq 0}(1+t)^{1 / 2}\left\|w_{\mathrm{h}}(t)\right\|_{L^{1}}+\sup _{t \geq 0}(1+t)\left\|w_{\mathrm{h}}(t)\right\|_{L^{\infty}} \leq C\left\|w^{0}\right\|_{L^{1} \cap H^{2}}
$$

but if we now return to (63) we obtain the stronger estimate

$$
\left\|\mathcal{J}_{\mathrm{h}}(t)\right\|_{L^{1}} \leq C\left\|w^{0}\right\|_{L^{1} \cap H^{2}}^{2} \int_{0}^{t} \frac{1}{(1+t-s)^{2}} \frac{1}{(1+s)} \mathrm{d} s \leq C \frac{\left\|w^{0}\right\|_{L^{1} \cap H^{2}}^{2}}{(1+t)}
$$

which also holds for $(1+t)^{1 / 2}\left\|\mathcal{J}_{\mathrm{h}}(t)\right\|_{L^{\infty}}$. This concludes the proof.

We next consider the central component $w_{0}(t, x) \in \mathbb{R}$, and prove that it behaves asymptotically like a solution of a linear equation with suitably modified initial data.

Proposition 6.2. If the initial data $w^{0} \in L^{1}\left(\mathbb{R}^{n}\right) \cap H^{2}\left(\mathbb{R}^{n}\right)$ are sufficiently small, the central component of the solution $w$ of (21) satisfies

$$
\begin{aligned}
\left\|w_{0}(t)-\mathcal{M}_{00}(t, 0) w_{\infty}\right\|_{L^{1}}+(1+t)^{n / 2} \| w_{0}(t)- & \mathcal{M}_{00}(t, 0) w_{\infty} \|_{L^{\infty}} \\
& \leq \frac{C}{(1+t)^{\gamma}}\left(\left\|w^{0}\right\|_{L^{1}}+\left\|w^{0}\right\|_{H^{2}}\right),
\end{aligned}
$$


where $\gamma=\frac{n}{4}+\frac{1}{r}<1$ and $w_{\infty} \in L^{1}\left(\mathbb{R}^{n}\right) \cap L^{\infty}\left(\mathbb{R}^{n}\right)$ is defined by

$$
w_{\infty}=\left(w^{0}\right)_{0}+\int_{0}^{\infty} K_{0}(s, w(s), \nabla w(s), \Delta w(s)) \mathrm{d} s .
$$

Proof. Projecting (49) onto the central component, we find

$$
\begin{aligned}
w_{0}(t) & =\mathcal{M}_{0} \cdot(t, 0) w^{0}+\int_{0}^{t} \mathcal{M}_{0} \cdot(t, s) K(s, w, \nabla w, \Delta w) \mathrm{d} s+\int_{0}^{t} \mathcal{M}_{0} \cdot(t, s) H(s, w) \mathrm{d} s \\
& =: \mathcal{M}_{0} \cdot(t, 0) w^{0}+\mathcal{I}_{0}(t)+\mathcal{J}_{0}(t),
\end{aligned}
$$

where $\mathcal{M}_{0} .(t, s)=\left(\mathcal{M}_{00}(t, s), \mathcal{M}_{0 \mathrm{~h}}(t, s)\right)$. Our goal is to extract from $w_{0}(t)$ the leading contributions as $t \rightarrow+\infty$. The last term $\mathcal{J}_{0}(t)$ is clearly negligible in this limit. Indeed, using Proposition 6.1 and proceeding as in (58), (59), we obtain

$$
\left\|\mathcal{J}_{0}(t)\right\|_{L^{1}}+(1+t)^{n / 2}\left\|\mathcal{J}_{0}(t)\right\|_{L^{\infty}} \leq \frac{C}{1+t}\left\|w^{0}\right\|_{L^{1} \cap H^{2}} .
$$

The same estimate holds for the linear term $\mathcal{M}_{0 \mathrm{~h}}(t, 0)\left(w^{0}\right)_{\mathrm{h}}$, because $\mathcal{M}_{\mathrm{oh}}(t, 0)$ decays as fast as $(1+t)^{-1} \mathcal{M}_{00}(t, 0)$ by Proposition 4.4. Using the same remark and proceeding as in (52), (54), (55), we see that the integral term $\mathcal{I}_{0 \mathrm{~h}}(t):=$ $\int_{0}^{t} \mathcal{M}_{0 \mathrm{~h}}(t, s) K_{\mathrm{h}}(s, w, \nabla w, \Delta w) \mathrm{d} s$ also satisfies (64). So the only remaining terms are $\mathcal{M}_{00}(t, 0)\left(w^{0}\right)_{0}$ and

$$
\begin{aligned}
\mathcal{I}_{00}(t)= & \int_{0}^{t} \mathcal{M}_{00}(t, s) K_{0}(s, w, \nabla w, \Delta w) \mathrm{d} s \\
= & \int_{t / 2}^{t} \mathcal{M}_{00}(t, s) K_{0}(s, w, \nabla w, \Delta w) \mathrm{d} s \\
& +\int_{0}^{t / 2}\left(\mathcal{M}_{00}(t, s)-\mathcal{M}_{00}(t, 0)\right) K_{0}(s, w, \nabla w, \Delta w) \mathrm{d} s \\
& +\mathcal{M}_{00}(t, 0) \int_{0}^{\infty} K_{0}(s, w, \nabla w, \Delta w) \mathrm{d} s \\
& -\mathcal{M}_{00}(t, 0) \int_{t / 2}^{\infty} K_{0}(s, w, \nabla w, \Delta w) \mathrm{d} s \\
= & : \mathcal{I}_{01}(t)+\mathcal{I}_{02}(t)+\mathcal{I}_{03}(t)+\mathcal{I}_{04}(t) .
\end{aligned}
$$

Proceeding as in (52), (55), it is straightforward to verify that

$$
\left\|\mathcal{I}_{01}(t)\right\|_{L^{1}}+(1+t)^{n / 2}\left\|\mathcal{I}_{01}(t)\right\|_{L^{\infty}} \leq \frac{C}{(1+t)^{\gamma}}\|w\|_{Y}^{2}, \quad t \geq 0
$$

where $\gamma=\frac{n}{4}+\frac{1}{r}<1$, and the same estimate clearly holds for $\mathcal{I}_{04}(t)$ too. Finally, using Remark 4.6 to bound the difference $\mathcal{M}_{00}(t, s)-\mathcal{M}_{00}(t, 0)$, we obtain

$$
\begin{aligned}
\left\|\mathcal{I}_{02}(t)\right\|_{L^{1}}+(1+t)^{n / 2}\left\|\mathcal{I}_{02}(t)\right\|_{L^{\infty}} & \leq C \int_{0}^{t / 2} \frac{s}{t}\left\|K_{0}(s, w, \nabla w, \Delta w)\right\|_{L^{1}} \mathrm{~d} s \\
& \leq \frac{C}{(1+t)^{\gamma}}\|w\|_{Y}^{2} .
\end{aligned}
$$

This concludes the proof of Proposition 6.2 because $\mathcal{M}_{00}(t, 0)\left(w^{0}\right)_{0}+\mathcal{I}_{03}(t)=$ $\mathcal{M}_{00}(t, 0) w_{\infty}$. 
It is now rather easy to prove Theorem 1.4 (in the case where $n \leq 3$ ). Combining Propositions 6.1 and 6.2, we find

$\left\|w(t)-e_{1} W(t)\right\|_{L^{1}}+(1+t)^{n / 2}\left\|w(t)-e_{1} W(t)\right\|_{L^{\infty}} \leq \frac{C}{(1+t)^{\gamma}}\left(\left\|w^{0}\right\|_{L^{1}}+\left\|w^{0}\right\|_{H^{2}}\right)$,

where $W(t)=\mathcal{M}_{00}(t, 0) w_{\infty}$ and $e_{1}=(1,0)^{\top}$ is the first vector of the canonical basis in $\mathbb{R}^{N}$. Furthermore, we claim that

$$
\left\|t^{n / 2} W\left(t, x t^{1 / 2}\right)-\tilde{\alpha} G\right\|_{L^{1} \cap L^{\infty}} \underset{t \rightarrow+\infty}{\longrightarrow} 0
$$

where $G$ is defined in (7) and

$$
\tilde{\alpha}=\int_{\mathbb{R}^{n}} w_{\infty}(x) \mathrm{d} x=e_{1}^{\top}\left(\int_{\mathbb{R}^{n}} w^{0}(x) \mathrm{d} x+\int_{0}^{\infty} \int_{\mathbb{R}^{n}} K(t, w, \nabla w, \Delta w) \mathrm{d} x \mathrm{~d} t\right) .
$$

To prove the $L^{\infty}$ claim in (66), we use Fourier transforms and simply note that the quantity

$$
\left\|\hat{W}\left(t, k t^{-1 / 2}\right)-\tilde{\alpha} \hat{G}(k)\right\|_{L^{1}}=\left\|M_{00}\left(t, 0 ; k t^{-1 / 2}\right) \hat{w}_{\infty}\left(k t^{-1 / 2}\right)-e^{-d_{0} k^{2}} \hat{w}_{\infty}(0)\right\|_{L^{1}}
$$

converges to zero as $t \rightarrow \infty$ by Lebesgue's dominated convergence theorem, in view of Proposition 4.1 and Remark 4.2. The $L^{1}$ claim can be established in a similar way, using the same ideas as in the proof of Proposition 4.4 (we omit the details).

We now return to the original variables. Since $u_{*}(t)=\Psi\left(\omega t e_{1}\right)$, the solution of (11) given by $u(t, x)=\Psi(v(t, x))=\Psi\left(v_{*}(t)+w(t, x)\right)$ can be decomposed as in (8), with $\alpha(t, x)=\omega^{-1} W(t, x)$ and

$$
\begin{aligned}
\beta(t, x)= & \Psi\left(v_{*}(t)+w(t, x)\right)-\Psi\left(v_{*}(t)\right)-\Psi^{\prime}\left(v_{*}(t)\right) w(t, x) \\
& +\Psi^{\prime}\left(v_{*}(t)\right)\left(w(t, x)-e_{1} W(t, x)\right) .
\end{aligned}
$$

Estimates (65), (66) immediately give (9), (10) with $\alpha_{*}=\omega^{-1} \tilde{\alpha}$. Finally, the formula (11) for $\alpha_{*}$ follows from the expression (67) of $\tilde{\alpha}$ and the fact that $U_{*}(0)=$ $\left(\Psi^{\prime}(0)^{-1}\right)^{\top} e_{1}, u_{*}^{\prime}(0)=\omega \Psi^{\prime}(0) e_{1}$. This concludes the proof of Theorem 1.4

\section{EXAMPLES AND PERSPECTIVES}

In this final section, we first give a simple example of a 2 -species reaction-diffusion system with a periodic orbit $u_{*}(t)$ which is asymptotically stable for the ODE dynamics but does not satisfy Hypothesis 1.2. We then discuss possible generalizations of the results of this paper.

7.1. Destabilization by diffusion: a simple example. One may feel inclined to believe that ODE-stable periodic orbits tend to be stable for the PDE dynamics, that is, that our Hypothesis 1.2 is satisfied in most cases where the periodic orbit is stable for the ODE. Our example below shows that this is not the case, even for a simple reaction-diffusion system with only two species.

We consider the following 2-species reaction-diffusion system:

$$
u_{t}=D u_{x x}+J u+\left(\epsilon^{2}-|u|^{2}\right) R u,
$$

where $u=\left(u_{1}, u_{2}\right)^{\top} \in \mathbb{R}^{2}$ and $|u|^{2}=u_{1}^{2}+u_{2}^{2}$. Here $\epsilon>0$ is a parameter, $D$ is a $2 \times 2$ real matrix with positive eigenvalues, and

$$
J=\left(\begin{array}{cc}
0 & -1 \\
1 & 0
\end{array}\right), \quad R=\left(\begin{array}{cc}
\cos (\theta) & -\sin (\theta) \\
\sin (\theta) & \cos (\theta)
\end{array}\right), \quad \theta \in(-\pi / 2, \pi / 2)
$$


System (68) has a $2 \pi$-periodic solution $u_{*}(t)=\epsilon \bar{u}(t)$, where $\bar{u}(t)=(\cos (t), \sin (t))^{\top}$. Linearizing (68) at $u_{*}(t)$ we obtain

$$
v_{t}=D v_{x x}+\left(J-2 \epsilon^{2} R \bar{u}(t) \bar{u}(t)^{\top}\right) v,
$$

or equivalently

$$
v_{t}=-k^{2} D v+\left(J-2 \epsilon^{2} R \bar{u}(t) \bar{u}(t)^{\top}\right) v, \quad k \in \mathbb{R} .
$$

Of course $v(t)=\bar{u}^{\prime}(t)$ is a solution of (69) for $k=0$.

Let $F=F_{0}(2 \pi, 0)$ be the Floquet matrix associated to (69) for $k=0$. Then

$$
\operatorname{Det}(F)=\exp \left(\operatorname{Tr} \int_{0}^{2 \pi}\left(J-2 \epsilon^{2} R \bar{u}(t) \bar{u}(t)^{\top}\right) \mathrm{d} t\right)=\exp \left(-2 \pi \epsilon^{2} \operatorname{Tr}(R)\right) .
$$

The Floquet exponents (for $k=0$ ) are therefore $\lambda_{1}=0$ and $\lambda_{2}=-\epsilon^{2} \operatorname{Tr}(R)$. As $\operatorname{Tr}(R)=2 \cos (\theta)>0$, it follows that $u_{*}(t)$ is a stable periodic orbit for the ODE dynamics associated to (68).

To compute the Floquet exponents for small $k$, we consider the adjoint ODE

$$
U_{t}=\left(J+2 \epsilon^{2} \bar{u}(t) \bar{u}(t)^{\top} R^{\top}\right) U .
$$

As is easily verified, the unique nontrivial bounded solution of (70) is $U_{*}(t)=$ $R \bar{u}^{\prime}(t)$. Using formula (4), we conclude that

$$
d_{0}=\frac{\int_{0}^{2 \pi} \bar{u}^{\prime}(t)^{\top} R^{\top} D \bar{u}^{\prime}(t) \mathrm{d} t}{\int_{0}^{2 \pi} \bar{u}^{\prime}(t)^{\top} R^{\top} \bar{u}^{\prime}(t) \mathrm{d} t}=\frac{\operatorname{Tr}\left(R^{\top} D\right)}{\operatorname{Tr}\left(R^{\top}\right)}=\frac{1}{2}(\operatorname{Tr}(D)-\tan (\theta) \operatorname{Tr}(J D)) .
$$

If the diffusion matrix $D$ is symmetric, then $\operatorname{Tr}(D)>0$ and $\operatorname{Tr}(J D)=0$; hence necessarily $d_{0}>0$, which means that the periodic solution $u_{*}(t)$ is spectrally stable for long wave-length perturbations. But if $D$ is a nonsymmetric matrix, then $\operatorname{Tr}(J D) \neq 0$ and therefore we can choose $\theta \in(-\pi / 2, \pi / 2)$ in such a way that $d_{0}<0$. This gives an example of a periodic orbit exhibiting a sideband instability.

On the other hand, as $\epsilon \rightarrow 0$, the periodic orbit $u_{*}(t)$ reduces to the fixed point $u=0$, for which it is easy to perform a stability analysis. For a fixed wavenumber $k \in \mathbb{R}$, we have to compute the eigenvalues $\lambda_{1}(k), \lambda_{2}(k)$ of the linearized operator $J-D k^{2}$. By direct calculation, we find

$\operatorname{Tr}\left(J-D k^{2}\right)=-\operatorname{Tr}(D) k^{2} \leq 0$ and $\operatorname{Det}\left(J-D k^{2}\right)=1+\operatorname{Tr}(J D) k^{2}+\operatorname{Det}(D) k^{4}$.

If we choose $D$ such that $\operatorname{Tr}(J D)+2(\operatorname{Det}(D))^{1 / 2}<0$, we see that there exists a nonempty open interval $I \subset(0,+\infty)$ such that $\operatorname{Tr}\left(J-D k^{2}\right)<0$ and $\operatorname{Det}\left(J-D k^{2}\right)<$ 0 if $k^{2} \in I$. Thus, one of the eigenvalues $\lambda_{i}(k)$ is strictly positive, which means that the equilibrium $u=0$ is unstable with respect to perturbations with wavenumbers $k$ such that $k^{2} \in I$. By continuity, this Turing instability persists for the periodic orbit $u_{*}(t)=\epsilon \bar{u}(t)$ if $\epsilon>0$ is sufficiently small: for $k^{2} \in I$, one of the Floquet exponents has a positive real part.

Summarizing, the periodic solution $u_{*}(t)=\epsilon \bar{u}(t)$ of the reaction-diffusion system (68) exhibits:

(1) a sideband instability if $\operatorname{Tr}(D)-\tan (\theta) \operatorname{Tr}(J D)<0$;

(2) a Turing instability if $\operatorname{Tr}(J D)+2(\operatorname{Det}(D))^{1 / 2}<0$ and $\epsilon \ll 1$. 


\section{Remarks 7.1.}

1. The instability criteria above are never satisfied if the matrix $D$ is symmetric. But if $D$ has eigenvalues $d_{1}>d_{2}>0$, we can choose an invertible matrix $S$ so that $S^{-1} D S=\mathcal{D}=\operatorname{diag}\left(d_{1}, d_{2}\right)$. Then setting $u=S w$ we obtain the equivalent system

$$
w_{t}=\mathcal{D} w_{x x}+S^{-1} J S w+S^{-1}\left(1-|S w|^{2}\right) R S w,
$$

where now the diffusion matrix has the usual diagonal form. The Floquet exponents characterizing the stability properties of the periodic orbit are of course unaffected by this linear transformation. Thus we can find 2-species systems of the form (71), with diagonal diffusion matrix, which exhibit either a sideband or a Turing instability.

2. Our example is clearly reminiscent of the complex Ginzburg-Landau equation (CGLE),

$$
u_{t}=(1+i a) \Delta u+u-(1-i c)|u|^{2} u
$$

where $a, c$ are real parameters and $u: \mathbb{R}_{+} \times \mathbb{R}^{n} \rightarrow \mathbb{C}$. Equation (72) possesses a homogeneous time-periodic solution of the form $u(t, x)=\mathrm{e}^{i c t}$, which exhibits a sideband instability if $a c>1$ (Benjamin-Feir criterion) and a Turing instability in other parameter regions; see e.g. [1]. The complex Ginzburg-Landau equation arises as a modulation equation near Hopf bifurcations in reaction-diffusion systems (see, for example, [29, 19]). One therefore expects stability and instability properties of small-amplitude periodic solutions near Hopf bifurcation to be governed by those of the CGLE; see, for example, 24] for a result in this direction. Since the diffusion matrix of CGLE possesses complex eigenvalues, it cannot be cast as a real reactiondiffusion system with diagonal diffusion matrix. Our example and the remark above show that one can almost explicitly recover the properties of CGLE with diagonal diffusion matrices. Upon substituting the diffusion matrix of CGLE in our example, one would recover precisely the Benjamin-Feir criterion from the instability criterion $\operatorname{Tr}(D)-\tan (\theta) \operatorname{Tr}(J D)<0$. In a different direction, one could also extend our results to diffusion matrices with $D+D^{\top}>0$ without any additional difficulties, which would then include CGLE as a particular example. In the context of reactiondiffusion modeling, cross-diffusion phenomena that are associated with off-diagonal elements of $D$ are however quite uncommon.

7.2. Discussion and perspectives. We believe that the method presented here can be adapted to other situations. We mention the stability of wave trains, $u(k x-\omega t)$, with $u(\xi)=u(\xi+2 \pi)$ and $\omega, k>0$, and Turing patterns $u(k x)=$ $u(-k x)$, with $u(\xi)=u(\xi+2 \pi)$ and $k>0$. In both cases, one finds a continuous spectrum with diffusive decay properties for the linearization. In both cases, the absence of relevant, self-coupling terms in the neutral mode has been shown previously; see [28, 9 .

Interesting questions arise when one attempts to extend the class of allowed perturbations. One may for instance consider perturbations $v$ such that $\nabla v \in L^{1}$. In one space-dimension, this would correspond to perturbations with different phase shifts at $x= \pm \infty$. One would still expect the diffusive linear part to be dominant so that one would find error function asymptotics for the phase correction. 
In fact, one would expect some type of stability for much more general perturbations. For instance, in one space-dimension, the homogeneous oscillation $u_{*}(t)$ is embedded in a family of wave train solutions $u(k x-\omega t ; k)$, with $k \approx 0$ and $u \approx u_{*}$; see [27, Section 3.3]. Under our stability assumptions, using Lyapunov-Schmidt reduction, one finds $\omega=\omega(k)=\omega_{0}+\omega_{2} k^{2}+\mathrm{O}\left(k^{4}\right)$; see for instance [22, Lemma 2.1]. Of course, $u(k x-\omega t ; k)$ is not close to any fixed homogeneous oscillation, but it is close to an appropriate phase shift of the oscillation in any finite region of space. All solutions in the basin of attraction of such wave trains then stay close to our homogeneous oscillation, orbitally, and pointwise in space. A natural question then asks for the asymptotics of initial conditions of the type $u(k(x) x-\omega(k(x)) t ; k(x))$, where $k(x) \rightarrow k_{ \pm}$for $x \rightarrow \pm \infty$. In the case of the real Ginzburg-Landau equation, which exhibits spatial oscillations $u(k x)$, this question was addressed in [7, 2, 13, showing that asymptotics are governed by a nonlinear diffusion equation $\theta_{t}=W\left(\theta_{x}\right)_{x}$, with locally uniform convergence to a fixed, intermediate wavenumber. Near temporal oscillations, one expects dynamics to be governed by a viscous conservation law $\theta_{t}=d\left(\theta_{x}\right)_{x}+j\left(\theta_{x}\right)$, so that solutions with asymptotically constant wavenumber would be expected to converge to viscous shocks [9, Section 8], or to rarefaction waves.

More generally, one could ask about the orbital stability of a family of oscillations: starting with an initial condition $u(k(x) x-\omega(k(x)) t ; k(x))$, with $k(x)$ bounded and $k^{\prime}$ small, will the solutions remain close to a solution of that form for all times? Again, this question possesses a simple answer for the approximation of the dynamics by a viscous conservation law due to the maximum principle, which gives immediate supremum bounds on $\theta_{x}$ in terms of the initial condition. For the full reaction-diffusion dynamics, however, which are only approximately described by a viscous conservation law, this question remains wide open.

\section{ACKNOWLEDGMEnTs}

The authors thank Sylvie Monniaux for useful discussions on maximal regularity in parabolic equations. The second author would like to thank the Université de Franche-Comté for generous support and hospitality during his stay, where part of this project was carried out. He also acknowledges partial support by the NSF through grant DMS-0504271.

\section{REFERENCES}

1. I. Aranson and L. Kramer. The world of the complex Ginzburg-Landau equation. Rev. Modern Phys. 74 (2002), 99-143. MR1895097 (2003h:35254)

2. J. Bricmont and A. Kupiainen. Renormalization group and the Ginzburg-Landau equation. Comm. Math. Phys. 150 (1992), 193-208. MR1188504 (94b:35255)

3. J. Bricmont, A. Kupiainen, and G. Lin. Renormalization group and asymptotics of solutions of nonlinear parabolic equations. Comm. Pure Appl. Math. 47 (1994), 893-922. MR 1280993 (95i:35138)

4. J. Buck and E. Buck. Synchronous fireflies. Scientific American 234 (1976), 74-79.

5. C. Chicone. Ordinary differential equations with applications. Second edition. Texts in Applied Mathematics 34, Springer, New York, 2006. MR2224508 (2006m:34001)

6. P. Collet and J.-P. Eckmann. Solutions without phase-slip for the Ginsburg-Landau equation. Comm. Math. Phys. 145 (1992), 345-356. MR1162802 (93d:35151) 
7. P. Collet, J.-P. Eckmann, and H. Epstein. Diffusive repair for the Ginzburg-Landau equation. Helv. Phys. Acta 65 (1992), 56-92. MR1163235 (93b:76037)

8. M. Cross and P. Hohenberg, Pattern formation outside of equilibrium. Rev. Mod. Phys. 65 (1993), 851-1123.

9. A. Doelman, B. Sandstede, A. Scheel, and G. Schneider. The dynamics of modulated wave trains. Memoirs Amer. Math. Soc. 199 (2009). MR2507940

10. J.-P. Eckmann, C.E. Wayne, and P. Wittwer. Geometric stability analysis for periodic solutions of the Swift-Hohenberg equation. Comm. Math. Phys. 190 (1997), 173-211. MR.1484552 (98m:35180)

11. N. Fenichel. Asymptotic stability with rate conditions. II. Indiana Univ. Math. J. 26 (1977), 81-93. MR0426056 (54:14002)

12. H. Fujita, On the blowing up of solutions of the Cauchy problem for $u_{t}=\Delta u+u^{1+\alpha}$. J. Fac. Sci. Univ. Tokyo Sect. I 13 (1966), 109-124. MR0214914 (35:5761)

13. T. Gallay and A. Mielke. Diffusive mixing of stable states in the Ginzburg-Landau equation. Comm. Math. Phys. 199 (1998), 71-97. MR.1660215 (99m:35223)

14. A.K. Ghosh, B. Chance, and E.K. Pye. Metabolic coupling and synchronization of NADH oscillations in yeast cell populations. Arch. Biochem. Biophys. 145 (1971), 319-331.

15. D. Henry. Geometric theory of semilinear parabolic equations. Lecture Notes in Mathematics, 840. Springer-Verlag, Berlin-New York, 1981. MR610244 (83j:35084)

16. M. Hieber and S. Monniaux. Heat-kernels and maximal $L^{p}-L^{q}$-estimates: The non-autonomous case. J. Fourier Anal. Appl. 6 (2000), 467-481. MR1781089 (2001h:34084)

17. T. Kapitula. On the nonlinear stability of plane waves for the Ginzburg-Landau equation. Comm. Pure Appl. Math. 47 (1994), 831-841. MR.1280990 (95e:35200)

18. A. Katok and B. Hasselblatt. Introduction to the modern theory of dynamical systems. Encyclopedia of Mathematics and its Applications 54. Cambridge University Press, 1995. MR.1326374 (96c:58055)

19. A. Mielke. The Ginzburg-Landau equation in its role as a modulation equation. Handbook of dynamical systems, Vol. 2, 759-834, North-Holland, Amsterdam, 2002. MR.1901066 (2003f:37148)

20. A. Pazy, Semigroups of Linear Operators and Applications to Partial Differential Equations, Appl. Math. Sci. 44, Springer-Verlag, New-York, 1983. MR710486 (85g:47061)

21. J. Prüss and R. Schnaubelt. Solvability and maximal regularity of parabolic evolution equations with coefficients continuous in time. J. Math. Anal. Appl. 256 (2001), 405-430. MR1821747 (2002e:34094)

22. J.D.M. Rademacher and A. Scheel. Instabilities of wave trains and Turing patterns in large domains. Int. J. Bif. Chaos 16 (2007), 2679-2691. MR2355524 (2009b:37135)

23. E. Risler, Criteria for the stability of spatial extensions of fixed points and periodic orbits of differential equations in dimension 2. Phys. D 146 (2000), 121-136. MR1787408|(2002j:35182)

24. E. Risler, A direct computation of the phase stability criterion for spatially homogeneous time periodic solutions close to a Hopf bifurcation. Internat. J. Bifur. Chaos Appl. Sci. Engrg. 11 (2001), 2097-2103. MR1859191 (2002g:35027)

25. E. Risler, Generic instability of spatial unfoldings of almost homoclinic periodic orbits. Comm. Math. Phys. 216 (2001), 325-356. MR1814850(2002e:37129)

26. F. Rogister, K. Thornburg, L. Fabiny, M. Müller, and R. Roy. Power-Law Spatial Correlations in Arrays of Locally Coupled Lasers. Phys. Rev. Lett. 92 (2004), 093905.

27. B. Sandstede and A. Scheel. Defects in oscillatory media: Toward a classification. SIAM J. Appl. Dyn. Syst. 3 (2004), 1-68. MR2067899(2005f:37166)

28. G. Schneider. Diffusive stability of spatial periodic solutions of the Swift-Hohenberg equation. Comm. Math. Phys. 178 (1996), 679-702. MR1395210(97f:35172)

29. G. Schneider. Hopf bifurcation in spatially extended reaction-diffusion systems. J. Nonlinear Sci. 8 (1998), 17-41. MR.1604562 (99k:35021)

30. H. Uecker. Diffusive stability of rolls in the two-dimensional real and complex SwiftHohenberg equation. Comm. Partial Differential Equations 24 (1999), 2109-2146. MR1720762 (2001d:35023)

31. G. Veser, F. Mertens, A. S. Mikhailov, and R. Imbihl. Global coupling in the presence of defects: Synchronization in an oscillatory surface reaction. Phys. Rev. Lett. 71 (1993), 935938 . 
32. A.T. Winfree. The geometry of biological time. Biomathematics, 8. Springer-Verlag, BerlinNew York, 1980. MR572965 (82c:92003)

Institut Fourier, UMR CNRS 5582, BP 74, Université de Grenoble I, 38402 SaintMartin-D'HÈres, France

E-mail address: Thierry.Gallay@ujf-grenoble.fr

School of Mathematics, University of Minnesota, 206 Church Street S.E., MinneapoLis, Minnesota 55455

E-mail address: scheel@umn.edu 